This is a postprint version of the following published document:

Ahedo, E., et al. Macroscopic and parametric study of a kinetic plasma expansion in a paraxial magnetic nozzle, In: Plasma sources science and technology, 29(4), 045017, April 2020, 17 pp.

DOI: https://doi.org/10.1088/1361-6595/ab7855

(C) 2020 IOP Publishing Ltd.

Creative Commons Attribution Non-Commercial No Derivatives License 


\title{
Macroscopic and parametric study of a kinetic plasma expansion in a paraxial magnetic nozzle
}

\author{
E. Ahedo ${ }^{1}$, S. Correyero ${ }^{1}$, J. Navarro ${ }^{1}$, M. Merino ${ }^{1}$ \\ ${ }^{1}$ Equipo de Propulsión Espacial y Plasmas, Universidad Carlos III de Madrid, Leganés, 28911, Spain, \\ Email:scorreye@uc3m.es.
}

\begin{abstract}
:
A kinetic paraxial model of a collisionless plasma stationary expansion in a convergent-divergent magnetic nozzle is analyzed. Monoenergetic and Maxwellian velocity distribution functions of upstream ions are compared, leading to differences in the expansion only on second and higher-order velocity moments. Individual and collective magnetic mirror effects are analyzed. Collective ones are small on the electron population since only a weak temperature anisotropy develops, but they are significant on the ions all over the nozzle. Momentum and energy equations for ions and electrons are assessed based on the kinetic solution. The ion response is different in the hot and cold limits, with the anisotropic pressure tensor being relevant in the first case. Heat fluxes of parallel and perpendicular energies have a dominant role in the electron energy equations. They do not fulfill a Fourier-type law; they are large even when electrons are near isothermal. A crude electron fluid closure based on a constant diffusion-to-convective thermal energy ratio is shown equivalent to the much invoked polytropic law. Analytical dimensionless parameter laws are derived for the nozzle total electric potential fall and the downstream residual electron temperature. Electron confinement and related current control by a thin Debye sheath and a the semi-infinite divergent magnetic nozzle are compared.
\end{abstract}

\section{Introduction}

The understanding of the expansion of magnetically-guided plasmas into vacuum is crucial to improve the propulsive performances of a magnetic nozzle (MN) [1]. This constitutes the main acceleration stage of some electrodeless plasma thrusters, proposed for in-space electric propulsion, such as the helicon plasma thruster (HPT) [2-5], the electron cyclotron resonance thruster [6], the applied-field magnetoplasma dynamic thruster [7], and the variable specific impulse magnetoplasma rocket (VASIMR) [8]. Magnetized plasma expansions are also relevant in other fields of plasma physics, for example in plasma sources for material processing and manufacturing [9] or in astrophysical jets [10].

Magnetized plasma beam expansions have been investigated extensively during the past years, both theoretically [3,11-15] and in the laboratory [5,16-23]. Given the inherent difficulty of measuring downstream properties reliably in low-density plasma plumes, theoretical models and simulation codes with the capability of accurately predicting the plasma response in the MN become crucial to study these devices. In the realm of plasma models, several approaches exist. On the one side, fluid models tend to be computationally fast and can provide much insight on the main properties of the plasma two-dimensional(2D) expansion [13, 14,24]. However, for a near-collisionless plasma, the closure of the fluid model with equations for the heat fluxes is far from obvious, and requires kinetic information on the plasma state. The fluid closure is generally more 
critical for electrons, which constitute a confined species with modest particle and energy drifts along the MN; ions are supersonically accelerated by the MN and its internal energy matters only in particular devices [25]. Commonly-claimed isothermal or polytropic closures for collisionless electrons lack proper physical justification: the first case is just an ad hoc simplification while the second one must be understood as a phenomenological closure based on fitting experimental data. This data fitting shows electron cooling in a divergent MN with a polytropic coefficient in the range 1.1-1.3 [26-29].

Paraxial (i.e. quasi one-dimensional) MN models, relying on the Vlasov equation, are showing to be an affordable and fruitful way to analyze the exact kinetic expansion of a collisionless plasma beam. In this context, Martínez-Sánchez et al. [30] developed a steady-state model computing self-consistently the velocity distribution function (VDF) of ions and electrons along a slender convergent-divergent MN. It was demonstrated that the downstream electron cooling and temperature anisotropy are a direct consequence of the emptying of regions of the velocity space of the electron VDF and that cooling determines the finite ambipolar potential drop along the MN.

The present paper aims to complete the analysis of Ref. [30] in several directions, part of them suggested from other, more recent works. In order of relevance, the first goal here is to analyze the equivalent fluid model of the MN. The solution of the Vlasov model of the paraxial MN is the VDF of ions and electrons along the nozzle. The successive integral moments in the velocity space of these VDFs provide the macroscopic plasma magnitudes, and the integral moments of the Vlasov equations they fulfill. This will allow, for instance, to assess the relevance, for ions and electrons, of (a) heat fluxes in the energy equations and (b) collective magnetic mirror effects in the momentum equations. The analysis of the equivalent fluid model was carried out partially by Sánchez-Arriaga, Zhou, et al. [31,32] within a paraxial, time-dependent kinetic model of a divergent-only $\mathrm{MN}$, finding out that electron heat fluxes were of the same order of magnitude than convective fluxes of electron thermal energy.

The second goal is to obtain parametric scaling laws of relevant plasma magnitudes, such as the total potential fall along the nozzle, or the downstream temperatures and heat fluxes. These laws are important for quick estimates of a MN performance without determining the full plasma response. Ramos et al. [33] carried out an analytical study of the asymptotic downstream response except for the heat fluxes.

The third objective is a detailed comparison of the different plasma response in the convergent and divergent regions of the MN, and the determination of the plasma conditions at the MN throat. Special attention will be given to the collective magnetic mirror effect on ions and electrons in each region and its coupling with the development of temperature anisotropy. Also, it will be shown that the ion VDF at the MN throat differs largely with the upstream ion VDF. This fact must be taken in to account when comparing convergent-divergent MN models with divergence-only MN models such as those of Refs. [31,34]. In particular, the comparison of the present model with the convergent-only MN of Ref. [35] will be addressed. The convergent-only MN model ends in a wall at a throat preceded by a thin Debye sheath. The similarities and differences between the plasma properties across this sheath and those across the whole divergent MN will be commented.

The last goal is to compare the plasma expansion for upstream monoenergetic and Maxwellian ion VDFs. Only the first case was considered in Ref. [30]. Velocity dispersion and heat fluxes are supposed to have a different importance in the two cases, mainly if the ion population is hotter than the electron one in the upstream source.

The paper is organized as follows. Section II summarizes the key aspects of the kinetic MN model. Section 
III presents spatial results, discusses the two cases of ion VDFs, compares the physics within the convergent and divergent regions, and presents parametric results at the MN throat and far downstream. Section IV discusses the macroscopic plasma equations, with special attention to the collective magnetic mirror effect and the relevance and modeling of the heat fluxes.

\section{Model formulation}

The kinetic MN model described in Ref. [30] is summarized here for self-containment. A convergentdivergent externally applied magnetic field $B(z)$ with a single maximum $B_{M}$ located at $z=0$ and vanishing at $z \rightarrow \pm \infty$ (where $B \rightarrow 0$ ) creates a MN that channels a fully magnetized ion-electron plasma generated at a source located at $z \rightarrow-\infty$. Subscripts ' 0 ', 'M', and ' $\infty$ ' will be used for locations $z \rightarrow-\infty$, $z=0$, and $z \rightarrow \infty$, respectively. The kinetic model attempts to determine the steady state of the paraxial, collisionless, quasineutral, current-free, low-beta, and fully-magnetized plasma plume in the MN. In this asymptotic limit, particle drifts and induced magnetic field effects are negligible. This ideal fully magnetized model would fail of course, at $|z|$ large enough where ion and electron magnetization fades, but this fact is marginal to the goals of the present study, centered on understanding basic plasma phenomena of the MN expansion.

Since the spatial distance $z$ does not appear explicitly in the model, a monotonic variable related directly to the non-monotonic magnetic field provides a more universal description of the spatial behavior. A convenient, space-like dimensionless variable is

$$
\zeta=\operatorname{sign}(z) \log _{10} \frac{B_{M}}{B}
$$

which places the throat $\mathrm{M}$ at $\zeta=0$ and scales logarithmically with $B$.

The model will determine iteratively the ambipolar electric potential $\phi(\zeta)$ and the VDFs of electrons and ions along the central magnetic line of the MN. It is assumed (and then confirmed by the solution) that $\phi(\zeta)$ decreases monotonically along the expansion from $\phi(-\infty)=0$ to $\phi(+\infty)=\phi_{\infty}(<0)$; this finite total potential fall along the $\mathrm{MN}$ is to be determined too.

As a consequence of full-magnetization and lack of collisions, electrons and ions conserve their total energy $E$ and magnetic moment $\mu$, that is

$$
E=\frac{m}{2}\left(w_{\|}^{2}+w_{\perp}^{2}\right)+e Z \phi, \quad \mu=\frac{m w_{\perp}^{2}}{2 B},
$$

where: species subscripts have been omitted, $m$ and $Z$ represent, respectively, the particle mass and charge number, $w_{\|}$is the particle velocity parallel to $B$ (i.e. axial in this model), and $w_{\perp}$ is the perpendicular velocity. Solving equation (2) for the two velocity components yields

$$
\begin{gathered}
w_{\perp}(\zeta, \mu)=\sqrt{\frac{2 \mu B(\zeta)}{m}}, \\
w_{\|}(\zeta, E, \mu)= \pm \sqrt{\frac{2}{m}[E-e Z \phi(\zeta)-\mu B(\zeta)]},
\end{gathered}
$$

where $w_{\perp}$ is positive by definition, while the plus and minus signs for $w_{\|}$indicate the forward and backward marching of particles, respectively.

Condition $w_{\|}(\zeta, E, \mu)=0$, i.e.

$$
E=Z e \phi(\zeta)+\mu B(\zeta)
$$



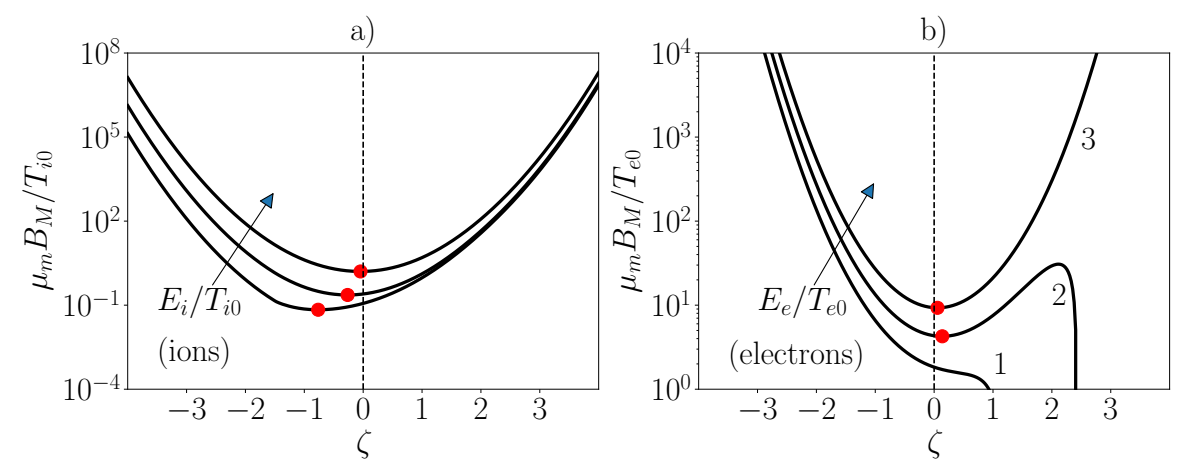

Figure 1: Three typical curves of $\mu_{m}$ for three ion and three electron energies (these ones increasing from curve 1 to 3). The red dots correspond to local minima $\mu_{T}(E)$. This example corresponds in fact to the solution for $m_{i} / m_{e}=10^{4}$ and $T_{i 0} / T_{e 0}=10$.

sets the spatial turning point for particles of given $(E, \mu)$; thus $e Z \phi+\mu B$ acts as the effective potential function for the parallel motion of a particle. Alternatively, equation (5) defines the maximum magnetic moment $\mu_{m}$ allowable for a particle with energy $E$ to reach location $\zeta$ :

$$
\mu_{m}(\zeta, E)=\frac{E-e Z \phi(\zeta)}{B(\zeta)}
$$

Figure 1(a)-(b) displays three typical spatial profiles of $\mu_{m}$ for ions $(i)$ and electrons $(e)$ and three different energies $E$. The curves show all the possible topologies found with the model discussed here.

The axial electric field pushes ions downstream and electrons upstream in both the convergent and divergent side of the nozzle. The magnetic mirror effect, in contrast, tends to keep both types of particles away from the throat region. This leads to different behaviors (a) of ions and electrons, and (b) in the convergent and divergent sides of the MN. Taking first the case of ions, for a given energy $E$ in figure 1(a), the curve $\mu_{m}(\zeta)$ always has a single minimum $\mu_{T}$ (red dot in the figure) at $\zeta=\zeta_{T}$ in the convergent side. Ions from the upstream source with low enough $\mu$ can surpass that location and therefore are free ions reaching $\zeta=+\infty$. The rest of them are reflected back to the source. Therefore, for given $E$, there are four $\mu$-subregions in the ion phase space: $\mu<\mu_{T}$, for free ions; $\mu_{T}<\mu<\mu_{m}$ and $\zeta<\zeta_{T}$, for reflected ions; $\mu_{T}<\mu<\mu_{m}$ and $\zeta>\zeta_{T}$, devoid of (upstream) ions; and $\mu>\mu_{m}$, forbidden energetically.

For electrons the situation is more complex. First, electrons with $E>e\left|\phi_{\infty}\right|$, such as those of curve 3 of figure 1(b), are free electrons, reaching $\zeta=+\infty$, or are reflected back to the source, depending on their magnetic moment $\mu$. Second, electrons with $E<e\left|\phi_{\infty}\right|$ lead to curves of type 1 or 2 of figure 1(b), and, if originated in the upstream source, are reflected back to it. In addition, for curves of type 2, with two local extrema, there is region of doubly-trapped electrons, bouncing between two locations of the divergent side: they are decelerated axially by $\phi$ when moving downstream, and by $B$ when moving upstream. The populations of reflected plus doubly-trapped electrons constitute the confined electron population.

As boundary conditions for the VDFs, it is assumed that there are no sources of particles at the downstream end, $\zeta=+\infty$, while, at the upstream plasma reservoir, the forward marching VDFs (i.e. with $w_{\|}>0$ and named $f^{+}$) of ions and electrons are known and semi-Maxwellian (except for a monoenergetic case discussed in Sec III), that is

$$
f_{0}^{+}(E)=n_{\star}\left(\frac{m}{2 \pi T_{\star}}\right)^{3 / 2} \exp \left(-\frac{E}{T_{\star}}\right)
$$


with $n_{\star}$ and $T_{\star}$ reference values of density and temperature. The backward-marching side of each VDF for reflected particles, named $f^{-}$, is to be determined.

Macroscopic magnitudes of ions and electrons are obtained from the velocity-integral moments of their VDF. Integration is more conveniently carried out in the invariants space $(E, \mu)$, so that for any magnitude $\chi$ its velocity integral becomes

$$
\langle\chi\rangle(\zeta)=\frac{2 \pi B}{m^{2}} \iint d \mu d E \frac{\chi f(E, \mu)}{\left|w_{\|}(\zeta, \mu, E)\right|} .
$$

Since doubly-trapped electrons are disconnected from both the upstream and downstream ends, in this stationary, collisionless model, their VDF can only be postulated. Here, following Ref. [30], the VDF expression for the reflected electrons will be also used for doubly-trapped electrons, which means a full replenishment of the doubly-trapped region during the transient MN formation.

The main macroscopic magnitudes for each species are density, particle (parallel) flux, parallel pressure, perpendicular pressure, (parallel) heat fluxes of parallel and perpendicular energy, given, respectively, by

$$
\begin{gathered}
n \equiv\langle 1\rangle, \quad n u=\left\langle w_{\|}\right\rangle, \\
p_{\|}=n T_{\|}=n m\left\langle c_{\|}^{2}\right\rangle, \quad p_{\perp}=n T_{\perp}=n m\left\langle w_{\perp}^{2}\right\rangle / 2, \\
q_{\|}=\frac{m}{2}\left\langle c_{\|}^{3}\right\rangle=\frac{m}{2}\left\langle w_{\|}^{3}\right\rangle-\frac{m}{2} n u^{3}-\frac{3}{2} p_{\|} u, \\
q_{\perp}=\frac{m}{2}\left\langle w_{\perp}^{2} c_{\|}\right\rangle=\frac{m}{2}\left\langle w_{\perp}^{2} w_{\|}\right\rangle-p_{\perp} u .
\end{gathered}
$$

Here, species subscripts are dropped, $u$ is the (axial) macroscopic velocity of the species, $c_{\|}=w_{\|}-u$ is the diffusion velocity of each species, and $T_{\|}$and $T_{\perp}$ are parallel and perpendicular temperatures. The average pressure is defined as $p=\left(p_{\|}+2 p_{\perp}\right) / 3$ and similarly for the average temperature. The parallel heat flux of total energy is $q=q_{\|}+q_{\perp}$. Notice that: the heat fluxes are indeed diffusive fluxes of thermal energy while the convective fluxes of parallel and perpendicular thermal energy are $(3 / 2) p_{\|} u$ and $p_{\perp} u$; only free subpopulations contribute to parallel fluxes of particles and energy; and, in this paraxial model, focused in the MN centerline all perpendicular fluxes are zero, i.e. $\left\langle w_{\perp}\right\rangle=\left\langle w_{\perp}^{3}\right\rangle=\left\langle c_{\|}^{2} w_{\perp}\right\rangle=0$.

Using equation (8) with $\left\langle w_{\|}\right\rangle$the particle flows are straightforwardly determined:

$$
\frac{n u}{B}=\frac{n_{\star}}{\sqrt{2 \pi m} T_{\star}^{3 / 2}} \int_{E_{0}}^{\infty} d E \mu_{T}(E) \exp \left(-\frac{E}{T_{\star}}\right)
$$

with $E_{0}=0$ for ions and to $E_{0}=-e \phi_{\infty}$ for electrons. These flows are constant spatially, which implies that ${ }_{155}$ $1 / B$ is proportional to the effective cross-section area of the flow.

For the density profiles, taking into account that $f_{i}^{-}=0$ for free ions and $f_{i}^{-}=f_{i}^{+}$for reflected ions, the ion density satisfies

$$
n_{i}(B, \phi)=\frac{n_{i \star}}{\sqrt{\pi} T_{i \star}^{3 / 2}} \int_{0}^{\infty} \exp \left(-\frac{E}{T_{i \star}}\right)\left(\sqrt{E-e \phi}+\operatorname{sign}\left[\zeta_{i T}(E)-\zeta\right] \sqrt{E-e \phi-B \mu_{i T}(E)}\right) d E .
$$

Similarly, for electrons one has [30]

$$
\begin{aligned}
n_{e}(B, \phi)=\frac{n_{e \star}}{\sqrt{\pi} T_{e \star}^{3 / 2}} \int_{-e \phi_{\infty}}^{\infty} \operatorname{sign}\left[\zeta_{e T}(E)-\zeta\right] \exp \left(-\frac{E}{T_{e \star}}\right) \sqrt{E+e \phi-B \mu_{e T}(E)} d E & +n_{e \star} \exp \frac{e \phi_{\infty}}{T_{e \star}}\left(\frac{1+\operatorname{erf} \sqrt{\xi}}{2} e^{\xi}-\sqrt{\frac{\xi}{\pi}}\right)
\end{aligned}
$$


with, in the last term, $\xi(\phi)=e\left(\phi-\phi_{\infty}\right) / T_{e \star}$. Taking the limit $\zeta \rightarrow-\infty$ (i.e. $B \rightarrow 0$ ) in these expressions, the upstream densities correspond indeed to the reference values:

$$
n_{i 0}=n_{i *}, \quad n_{e 0}=n_{e *}
$$

It will later found that $T_{i 0}=T_{i *}$ and $T_{e 0}=T_{e *}$ too. This means that, at $\zeta=-\infty$, the back-marching VDFs of ions and electrons are practically semi-Maxwellian. In other words, at $\zeta \rightarrow-\infty$, the contribution of the free populations of ions and electrons is a set of measure zero on the velocity integral moments.

Hereafter, only the case of a current-free plasma beam is discussed, i.e. equal fluxes of ions and electrons in the MN. Then, the total potential drop $\left|\phi_{\infty}\right|$ and the profile of the ambipolar electric potential profile $\phi(\zeta)$ must be such that the plasma beam satisfies the current-free and quasineutrality conditions,

$$
e\left(n_{i} u_{i}-n_{e} u_{e}\right)=0, \quad n_{i}(B, \phi) \simeq n_{e}(B, \phi) \equiv n .
$$

The iteration procedure on $\phi(\zeta)$ to fulfill the above conditions and obtain the self-consistent solution of the problem is the same than in Ref. [30]. Notice that equations (16) states that in a paraxial current-free plasma $u_{e}=u_{i}$. The extension of the model to a current-carrying plasma is straightforward, just requiring to change one boundary condition.

Plasma equations are normalized with $B_{M}, n_{0}, T_{e 0}$, and $\sqrt{T_{e 0} / m_{i}}$. Then, the dimensionless model turns out to depend only on two parameters: the ion-to-electron temperature ratio and the ion-to-electron mass ratio, $T_{i 0} / T_{e 0}$ and $m_{i} / m_{e}$.

\section{Kinetic results}

\section{III.1 Spatial profiles and influence of the ion VDF form}

Reference [30] studied the MN model with Maxwellian electrons and monoenergetic ions with

$$
f_{i 0}^{+}(E)=\frac{n_{i 0} m_{i}^{3 / 2}}{4 \pi\left(3 T_{i 0}\right)^{1 / 2}} \delta\left(E-\frac{3}{2} T_{i 0}\right),
$$

with $\delta$ the Dirac function. Figure 2 compares the plasma expansion along the nozzle for the monoenergetic and Maxwellian ion VDFs. Figure 2(a) shows that the electric potential profiles $\phi(\zeta)$ are practically the same for both ion VDFs. This indicates that $\phi(\zeta)$ does not depend practically on the velocity dispersion of the ion VDF, what makes sense since it is determined from conditions (16) involving only plasma densities and flows. Section III.3 will further compare electric potential drops for the two ion VDFs. two ion VDFs, all electron macroscopic magnitudes are almost identical for both distributions, as figure 2(b), (c), (f), and (i) corroborate. Since ions and electrons share density and axial velocity, $n$ and $u_{i}$, differences on macroscopic variables between the two ion VDFs are noticeable only for higher velocity moments, such as ion temperatures and heat fluxes. This is well illustrated in figure $2(\mathrm{~d}),(\mathrm{e}),(\mathrm{g})$, and $(\mathrm{h})$.

Differences in $T_{i \|}$ are seen only in the divergent region. Downstream, $T_{i \|}$ goes to zero only for the monoenergetic distribution, while a reduced parallel velocity dispersion is kept for the Maxwellian one, i.e. $T_{i \| \infty} \neq 0$. On the contrary, $T_{i \perp}$ is the same for both distributions and goes to zero downstream $\left(T_{i \perp \infty}=0\right)$ due to the inverse magnetic mirror effect, as it will be discussed later. Whereas ions develop a significant temperature 

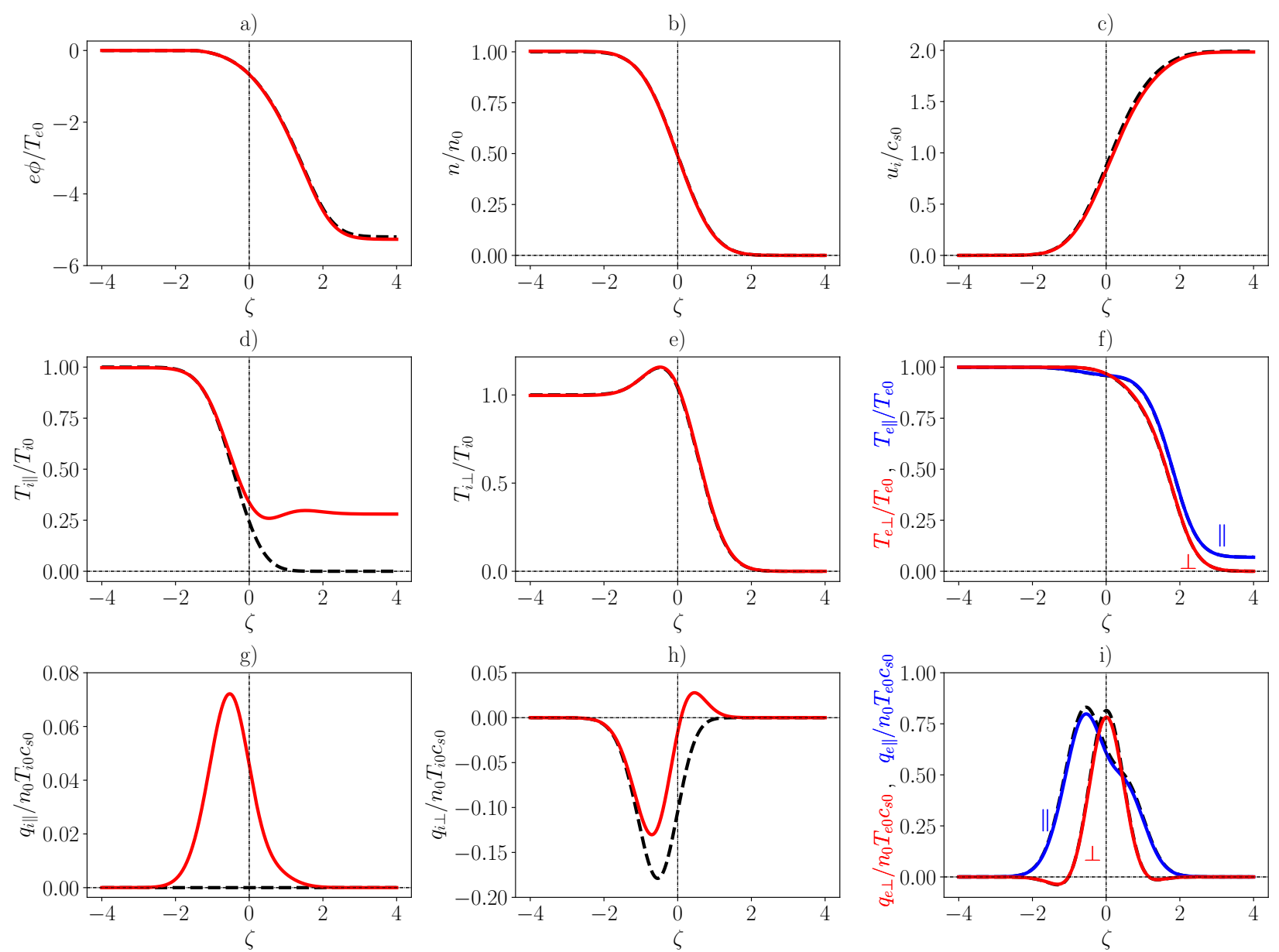

Figure 2: Normalized plasma properties for Maxwellian (— and —— ) and monoenergetic (--- ) ions, with $m_{i} / m_{e}=10^{4}$ and $T_{i 0} / T_{e 0}=10$.

anisotropy along the MN, the electron temperature is practically isotropic except far downstream. Ion and electron heat fluxes present complex behaviors that will be discussed in Section IV. Finally, the qualitative trends shown in figure 2 for the hot-ion case continue to be valid in the cold-ion case, i.e. for $T_{i 0} / T_{e 0} \ll 1$. Some parametric studies on the influence of $T_{i 0} / T_{e 0}$ and $m_{i} / m_{e}$ on the solution are developed below.

\section{III.2 Behavior on the convergent region and the MN throat}

The magnetic mirror effect (MME) on both ions and electrons makes the plasma response very different in the convergent side, where it slows down the parallel motion of individual ions and electrons, and the divergent side, where it accelerates axially ions and electrons. When this behavior is combined with the effect of the electric potential, $\phi(\zeta)$, which is similar in both nozzle sides but opposite for ions and electrons, it turns out that the collective MME is very different for ions and electrons.

Figure 3 plots the main plasma properties at the magnetic throat M. Plot 3(a) shows that the potential fall in the convergent side scales mainly with the electron temperature and lies within the interval

$$
-e \phi_{M} / T_{e 0} \sim 0.55-0.70
$$

if $m_{i} / m_{e}>10^{3}$ (that is for ion-electron plasmas). Figure 3(b) shows that the fraction of free electrons at the $\mathrm{MN}$ throat (and therefore in the convergent $\mathrm{MN}$ ) is still very small. The increase of that fraction when 
a)

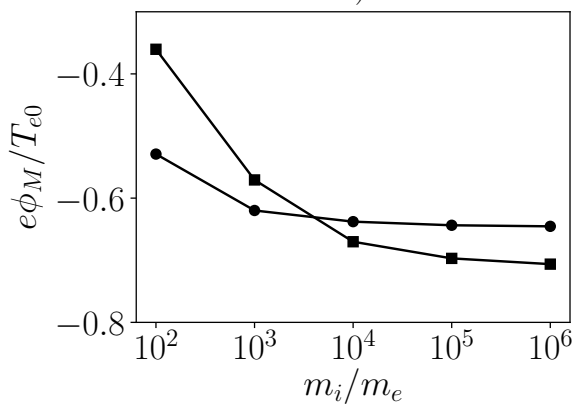

c)

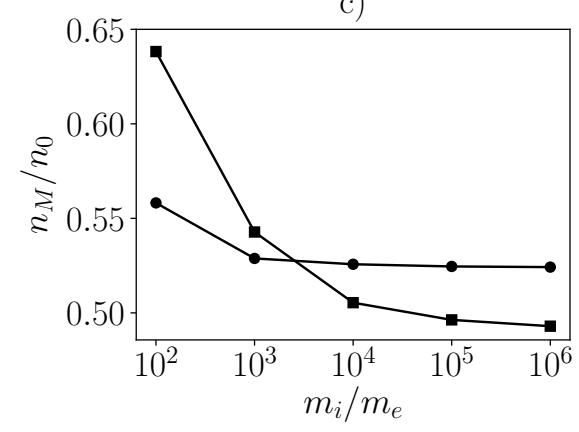

e)

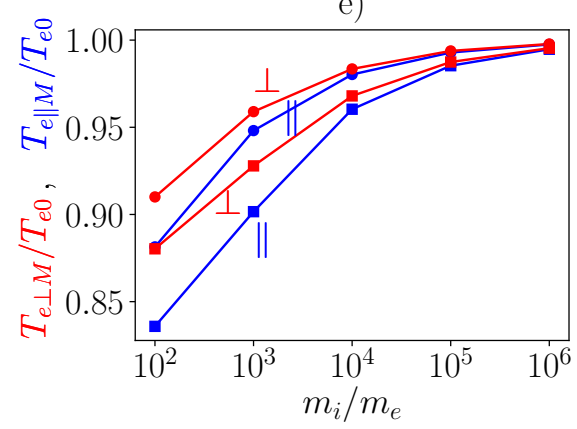

b)

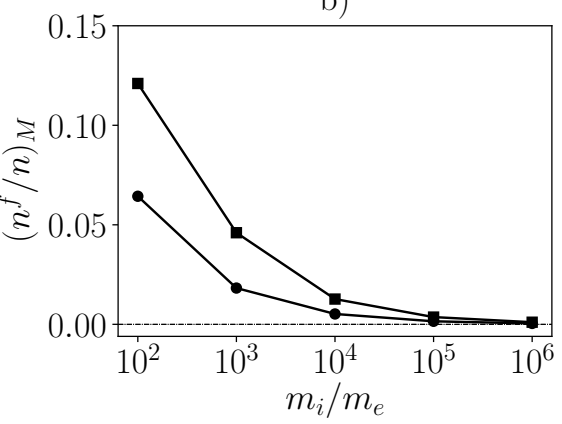

d)

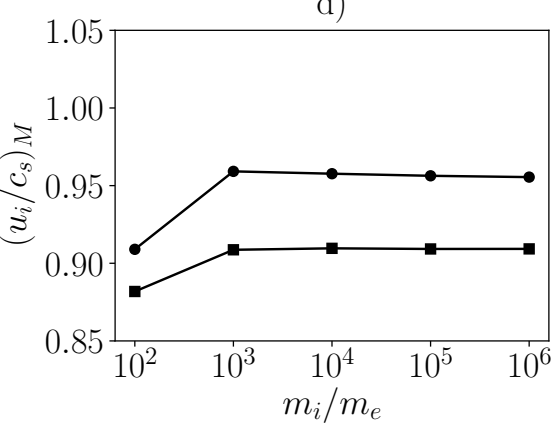

f)

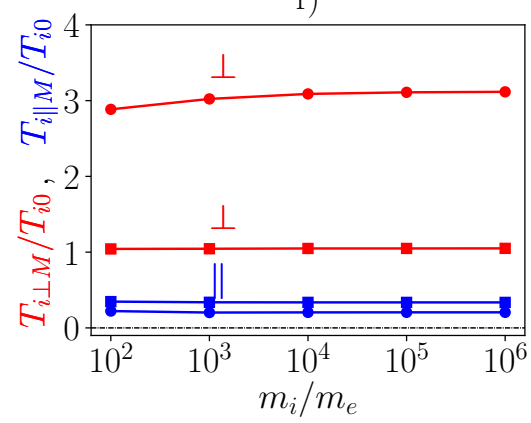

Figure 3: Normalized plasma properties at the magnetic throat $M$, for different $m_{i} / m_{e}$ and $T_{i 0} / T_{e 0}=0.1(\rightarrow-)$ and 10 (-). (a) Plasma potential, (b) ion velocity, (c) plasma density, (d) free electron density, (e) electron temperatures, and $(f)$ ion temperatures.

$m_{i} / m_{e}$ decreases is due to a lower electrostatic confinement of electrons, caused by a lower $\left|\phi_{\infty}\right|$. Figure 3(c) plots the plasma density at $\mathrm{M}$, which follows approximately the Boltzmann relation, $n_{M} / n_{\star} \simeq \exp \left(e \phi_{M} / T_{e 0}\right) \sim$ $0.50-0.54$; the next Section will explain this behavior. Figure 3(d) plots the ratio of the plasma velocity versus the reference sound speed at the throat, $c_{s M}=\sqrt{\left(T_{e M}+T_{i M}\right) / m_{i}}$. Notice that the plasma here is a collisionless fluid, and an expression for the real sound speed would require an exact closure of the fluid equations, which the next Section finds unavailable. Nonetheless, it is still reasonable to say that the plasma beam experiences a sonic transition around the nozzle throat.

Figures 3(e) and 3(f) show the parallel and perpendicular temperatures of ions and electrons at $M$. While electrons remain practically isothermal in the convergent $\mathrm{MN}$, ion temperature components behave differently and develop an anisotropy. In fact, although $T_{i \|}$ decreases similarly for both hot- and cold-ion simulations ( $T_{i \|} \simeq 0.2 T_{i 0}$ and $T_{i \|} \simeq 0.33 T_{i 0}$ for cold- and hot-ion cases, respectively), $T_{i \perp}$ increases at the throat. This increase is related to the existence of a low-velocity empty region in the ion VDF at the throat, of characteristic energy $\left|e \phi_{M}\right| \sim T_{e 0}$, as discussed in the Appendix A. This empty region becomes more relevant in the integral 
for $T_{i \perp}$ the lower the characteristic ion energy $T_{i 0}$. Therefore, the temperature anisotropy in the ion population is more marked in the case of initially cold ions, $T_{i 0} / T_{e 0} \ll 1$.

Both ion and electron particles suffer a similar a magnetic mirror effect, reducing the parallel particle velocity and reflecting back particles with $w_{\|}=0$. However, the collective MME is very different in the two VDFs populations, due to the different effect of the electric potential on the two species: additional confinement of electrons and parallel acceleration for ions. It is shown later that that the collective MME on a given species appears as the volumetric force $n\left(T_{\perp}-T_{\|}\right) d \ln B / d z$ in its momentum equation, so a collective MME is closely related to the development of temperature anisotropy. Therefore, figures 3(e) and (f) suggest the existence of a strong collective MME on the ions but a negligible one on the electrons. For electrons the individual magnetic mirror plus the electric potential make the VDF to decrease its density along the convergent MN, while remaining nearly Maxwellian and isotropic. Appendix A discusses and plots the evolution of the ion and electron VDFs on the convergent MN, in order to better understand their collective behaviors.

Several works on kinetic models consider the plasma expansion in a divergent MN only, placing the plasma source at the throat M (or nearby) [31,36]. This configuration makes full sense when plasma processes on the convergent MN are dominated by phenomena different from magnetic guiding, such as plasma production and heating or interaction with chamber walls. The plasma conditions imposed at the throat in these models generally differ partially with the present ones, for instance on the ion temperature anisotropy and the beam 'near-sonic' macroscopic velocity. This fact must be taken into account for a proper comparison with the present model.

\section{III.3 Parametric laws for downstream magnitudes}

The asymptotic downstream values of plasma magnitudes are important variables characterizing the plasma response in the MN. They depend on the two free parameters of the model: the mass ratio, $m_{i} / m_{e}$, which defines the propellant type; and the temperature ratio $T_{i 0} / T_{e 0}$, which species stores more internal energy upstream.

The main downstream variable is surely the total electric potential fall in the convergent-divergent $\mathrm{MN}$, plotted in Figure 4(a). The dimensionless final potential, $e\left|\phi_{\infty}\right| / T_{e 0}$, increases logarithmically with the mass ratio. For electron-ion plasmas -i.e. $m_{i} / m_{e} \geq O\left(10^{3}\right)$-, the potential fall (including the drop in the convergent $\mathrm{MN}$ ) is 5-8 times the upstream electron temperature, which agrees reasonably well with experimental data, taking into account the differences between this model and practical configurations [27,29]. The dimensionless final potential depends weakly on the temperature ratio: it almost does not change from $T_{i 0} / T_{e 0}=0.1$ to 1 , and only by a $15-20 \%$ percent from $T_{i 0} / T_{e 0}=1$ to 10 . This implies that $\left|\phi_{\infty}\right|$ is set mainly by the electron thermal energy and is consistent with the role of $\phi$ of confining most of the electron population. Approximate semi-analytical fittings for $e\left|\phi_{\infty}\right| / T_{e 0}$ versus $m_{i} / m_{e}$ and $T_{i 0} / T_{e 0}$ are derived below.

Figure $4(\mathrm{~b})$ plots the plasma beam velocity, For the cold-ion case $T_{i 0} / T_{e 0}=0.1$, the numerical linear fitting is

$$
\frac{u_{i \infty}}{\sqrt{T_{e 0} / m_{i}}} \approx 0.168 \ln \frac{m_{i}}{m_{e}}+1.95,
$$

and the two coefficients in this linear fitting become $(0.160,2.25)$ and $(0.118,4.40)$ for $T_{i 0} / T_{e 0}=1$ and 10 , respectively. In the cold-ion case, one has $u_{i \infty} \approx \sqrt{2 e\left|\phi_{\infty}\right| / m_{i}}$, while a relevant contribution from the conversion 
a)

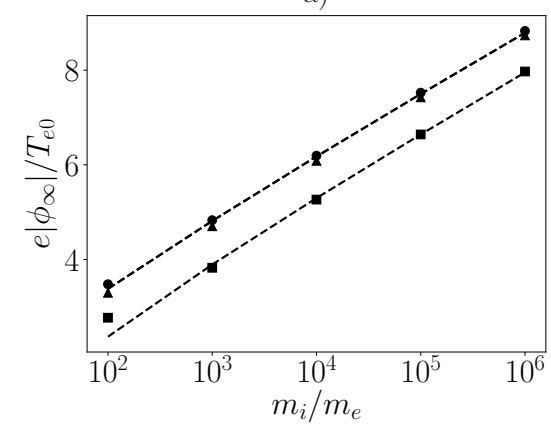

c)

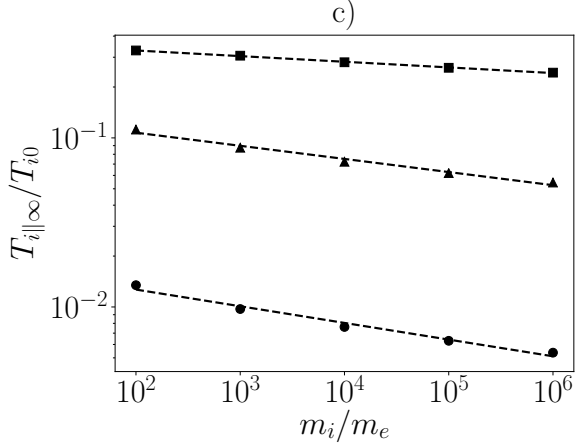

b)

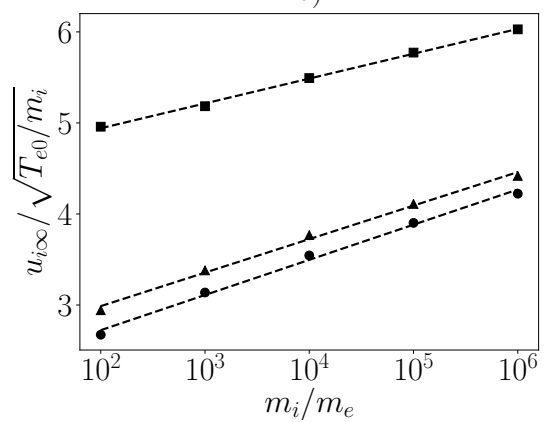

d)

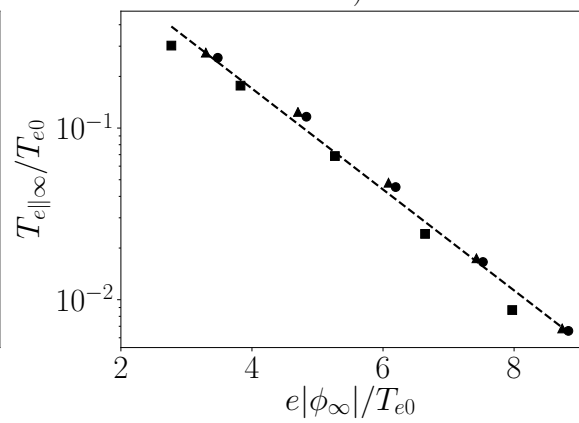

Figure 4: (a) Total dimensionless potential drop versus the mass ratio, (b) plasma beam velocity versus the mass ratio, (c) downstream parallel ion temperature versus the mass ratio and (d) downstream parallel electron temperature versos the total potential drop. $T_{i 0} / T_{e 0}=0.1(\bullet), T_{i 0} / T_{e 0}=1.0(\bullet)$ and $T_{i 0} / T_{e 0}=10(\bullet)$; the dashed lines correspond to approximate fitting laws.

of ion thermal energy into kinetic energy is added in the hot-ion cases. In plasma thrusters, the final beam velocity is closely related to the specific impulse, $I_{s p}$. To this respect it is interesting to observe that, to dominant order, $u_{i \infty} \propto \sqrt{m_{e} / m_{i}} \ln \left(m_{i} / m_{e}\right)$, so that the heavier the propellant is, the larger electric potential fall compensates partially the higher ion-mass penalty in $I_{s p}$.

Since $u_{i \infty}$ is finite, the continuity equation (13) states that the plasma density goes to zero as $n \propto B^{-1}$. The perpendicular temperatures of ions and electrons also go to zero, i.e. $T_{i \perp \infty}=T_{e \perp \infty}=0$, due to the conservation of the magnetic moment (i.e. the inverse magnetic mirror effect). However, the parallel velocities of ions and electrons keep part of their upstream dispersion and the respective downstream parallel temperatures of ions and electrons are not zero. Figure $4(\mathrm{c})$ plots $T_{i \| \infty} / T_{i 0}$. For the cold-ion case $T_{i 0} / T_{e 0}=0.1$, the numerical linear fitting is

$$
\frac{T_{i \| \infty}}{T_{i 0}} \approx-0.099 \ln \frac{m_{i}}{m_{e}}-3.91
$$

The two coefficients in this linear fitting become $(-0.077,-1.87)$ and $(-0.033,-0.96)$ for $T_{i 0} / T_{e 0}=1$ and 10 , respectively. Figure $4(\mathrm{~b})$ shows that the dependence of $T_{e \| \infty} / T_{e 0}$ on the mass and temperature ratios is through the final electric potential fall, via the numerical linear fitting

$$
\ln \frac{T_{e \| \infty}}{T_{e 0}}=-0.67 \frac{e\left|\phi_{\infty}\right|}{T_{e 0}}+0.89 .
$$

Both final parallel temperature ratios are much less than one and they are lower the higher $m_{i} / m_{e}$ and, thus $\left.e\left|\phi_{\infty}\right| / T_{e 0}\right)$ are. It can also be concluded that the final electric potential depends more on the electron state than on the ion one. 
a)

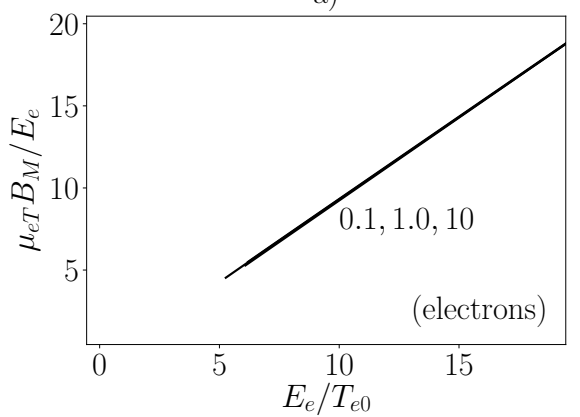

b)

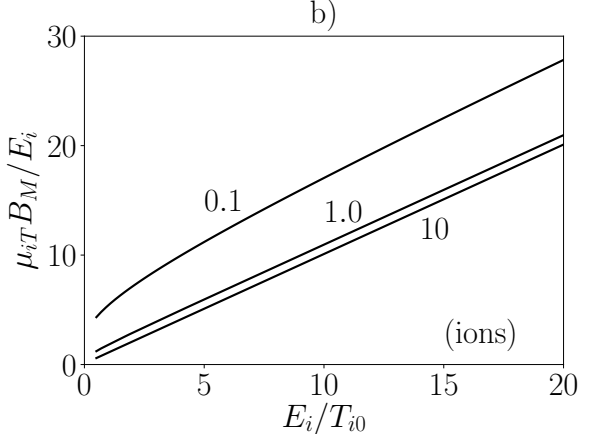

Figure 5: Functions $\mu_{T}(E)$ for ions and electrons, $T_{i 0} / T_{e 0}=0.1,1.0$ and 10. Plots are for $m_{i} / m_{e}=10^{4}$ but $\mu_{i T}(E)$ and $\mu_{e T}(E)$ are practically independent of $m_{i} / m_{e}$.

Next, Fig. 5 presents $\mu_{T}(E)$ for ions and electrons for the parametric ranges $m_{i} / m_{e}=10^{2}-10^{5}$ and $T_{i 0} / T_{e 0}=0.1-10$. As Ref. [30] showed and figure 5(a) illustrates, the electron function $\mu_{e T}(E)$ is practically independent of the two dimensionless parameters and satisfies rather accurately the linear relation

$$
\mu_{e T}(E) \approx E / B_{M}
$$

For Maxwellian ions, figure $5(\mathrm{~b})$ shows that $\mu_{i T}(E)$ is independent of the mass ratio, but depends on $T_{i 0} / T_{e 0}$. Only for the hot-ion case the approximation $\mu_{i T}(E) \approx E / B_{M}$ is applicable. Using these linear approximations for $\mu_{e T}(E)$ and $\mu_{i T}(E)$, analytical expressions are found for the flows of electrons and hot ions in equation (13). For mass ratios above $O\left(10^{2}\right)$ and Maxwellian VDFs, these are

$$
\frac{n u_{e}}{B} \simeq \frac{n_{e 0}}{B_{M}} \sqrt{\frac{T_{e 0}}{2 \pi m_{e}}}\left(\frac{e\left|\phi_{\infty}\right|}{T_{e 0}}+1\right) \exp \left(-\frac{e\left|\phi_{\infty}\right|}{T_{e 0}}\right)
$$

and

$$
\frac{n u_{i}}{B} \simeq \frac{n_{i 0}}{B_{M}} \sqrt{\frac{T_{i 0}}{2 \pi m_{i}}} \simeq 0.40 \frac{n_{i 0}}{B_{M}} \sqrt{\frac{T_{i 0}}{m_{i}}} \quad\left(\text { for } \frac{T_{i 0}}{T_{e 0}} \gg 1\right) .
$$

Equating both flows, the total potential fall satisfies the implicit equation

$$
\frac{e\left|\phi_{\infty}\right|}{T_{e 0}}-\ln \left(1+\frac{e\left|\phi_{\infty}\right|}{T_{e 0}}\right)=\ln \sqrt{\frac{m_{i} T_{e 0}}{m_{e} T_{i 0}}}-0.2, \quad \text { for } \quad \frac{m_{i}}{m_{e}} \gg \frac{T_{i 0}}{T_{e 0}} \gg 1,
$$

where the offset -0.2 has been added from the linear numerical fitting plotted in figure $4(\mathrm{a})$. Notice that the second term on the left side of equation (25) provides just a mild correction to the first one.

For the cold-ion case, no simple-enough expression for $\mu_{i T}(E)$ has been obtained, but it is found that the dimensionless potential fall depends no more on $T_{i 0}$. A suitable numerical fitting based on the results of figure $4(\mathrm{a})$ is

$$
\frac{e\left|\phi_{\infty}\right|}{T_{e 0}}-\ln \left(1+\frac{e\left|\phi_{\infty}\right|}{T_{e 0}}\right)=\ln \sqrt{\frac{m_{i}}{m_{e}}}-0.4, \quad \text { for } \quad \frac{T_{i 0}}{T_{e 0}} \ll 1 .
$$

These two fittings of the MN potential fall for the cold- and hot-ion cases match at $T_{i 0} / T_{e 0} \approx 1.5$.

Figure 2(a) showed that $\left|\phi_{\infty}\right|$ was very similar for Maxwellian and monoenergetic ion VDFs, for same mass and temperature ratio. Indeed, for a monoenergetic and hot-ion VDF, equation (17), the ion flow satisfies

$$
\frac{n u_{i}}{B} \simeq \frac{n_{i 0}}{B_{M}} \sqrt{\frac{3 T_{i 0}}{16 m_{i}}} \simeq 0.43 \frac{n_{i 0}}{B_{M}} \sqrt{\frac{T_{i 0}}{m_{i}}}, \quad \text { for } \quad \frac{m_{i}}{m_{e}} \gg \frac{T_{i 0}}{T_{e 0}} \gg 1,
$$

which differs very little from equation (24) for a Maxwellian VDF, thus justifying the previous observation. 
Figure 3(a) and equation (18) showed that the potential drop in the convergent MN is modest compared to the total potential drop in the MN. Hence, the above scaling laws for $e\left|\phi_{\infty}\right| / T_{e 0}$ can then be taken as valid approximations for the potential fall in the divergent nozzle, $e \phi_{\infty M} / T_{e 0}$, with $\phi_{\infty M}=\phi_{M}-\phi_{\infty}$. Then, the logarithmic dependence of $e \phi_{\infty M} / T_{e 0}$ on $m_{i} / m_{e}$ resembles much the one for the potential fall in a conventional Debye sheath next to a wall. To this respect, it is worth to compare the model for the magnetic cusp (indeed a convergent MN) studied by Martínez-Sánchez and Ahedo [35] to the present convergent-divergent MN one. In their case the plasma was current-free too, and the convergent MN was followed by a Debye sheath (of zero thickness and non-neutral) and a solid wall located at the MN throat, while here the convergent MN is followed by the divergent MN (of infinite extension and quasineutral).

The total potential fall within their sheath and the one within the present divergent MN have indeed the same role: to confine appropriately most of the electrons in order that the electron current leaking downstream is the correct one (i.e. equal to the ion current for a current-free beam). Electron and ion physics are shown here to be more complex in the divergent MN than in the planar, purely-electrostatic sheath, but the required potential drops for electron confinement are rather similar.

Figure 2 showed that most plasma magnitudes reach their downstream asymptotic values around $\zeta \sim 2$,

\section{Macroscopic plasma description}

\section{IV.1 The equivalent fluid model}

Taking velocity moments of the Vlasov equation for a generic VDF, the fluid equations for the bulk variables are obtained. For the present paraxial flow, the main fluid equations for each species are

$$
\begin{aligned}
& \frac{n u}{B}=\text { const, } \\
& B \frac{d}{d \zeta}\left(\frac{m n u^{2}}{B}\right)+n Z e \frac{d \phi}{d \zeta}+\frac{d p_{\|}}{d \zeta}-\left(p_{\|}-p_{\perp}\right) \frac{d \ln B}{d \zeta}=0, \\
& \frac{n u Z e \phi}{B}+\frac{n u}{B}\left[\frac{m u^{2}}{2}+\frac{3 T_{\|}}{2}+T_{\perp}\right]+\frac{q_{\|}+q_{\perp}}{B}=\text { const, } \\
& \frac{n u T_{\perp}+q_{\perp}}{B^{2}}=\text { const, }
\end{aligned}
$$

corresponding to the conservation of particles, momentum, total energy, and perpendicular energy, respectively. The constant in the continuity equation was already determined in equation (13). The momentum equation (28), which cannot be reduced to a first integral, shows $1 / B$ as the effective cross-section variation in the convective term, and includes the collective MME as a third volumetric force. The total energy conservation equation (29) includes the contributions of the kinetic and thermal energy flows plus the parallel heat flows of parallel and perpendicular energies, plus the 'flow of potential energy' (indeed, the integral of the work of the 

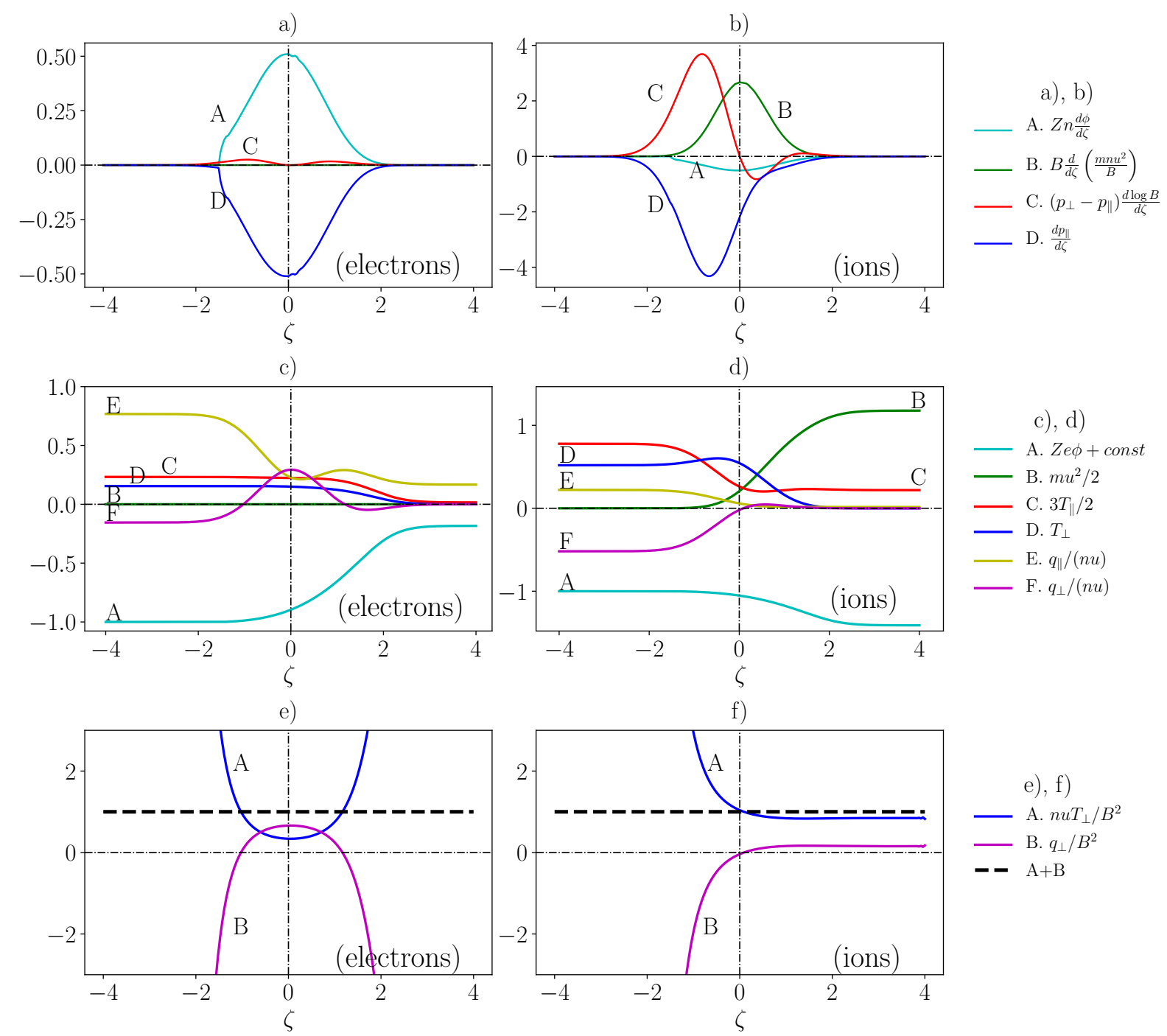

e), f)

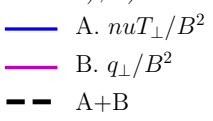

Figure 6: Relative contribution for electrons (left) and hot ions (right) of the different terms of the fluid equations of (a)-(b) momentum, (c)-(d) total energy per particle, and (e)-(f) perpendicular energy. Units in vertical axes are arbitrary. Results are for $m_{i} / m_{e}=10^{4}$ and $T_{i 0} / T_{e 0}=10$.

electric field). Dividing this equation by equation (27), the conservation of total energy 'per particle' in the ion or electron flow is

$$
\frac{m u^{2}}{2}+\frac{3 T_{\|}}{2}+T_{\perp}+\frac{q_{\|}+q_{\perp}}{n u}+Z e \phi=\mathcal{E},
$$

with $\mathcal{E}$ the total energy per (average) particle. Finally, the very simple form of the perpendicular energy equation (30), without kinetic energy flow and electric field work, is due to the paraxial approximation (i.e. it is the perpendicular energy in the centerline). The factor $1 / B^{2}$ in this equation combines the effect of the cross-section variation and the magnetic mirror effect (which increases the perpendicular kinetic energy proportionally to $B$ ).

Since these fluid equations are exact moments of the Vlasov equation they must be satisfied by the integral velocity moments of the ion and electron VDFs obtained directly from the kinetic model. In fact the error in fulfilling these fluid equations measures the error of the numerical integration algorithms; in all cases presented here it has been checked that this error is negligible. The analysis of the fluid terms for $m_{i} / m_{e}=10^{4}$ and $T_{i 0} / T_{e 0}=10$ is presented in Figure 6 for electrons (left) and ions (right). Each subplot depicts the different 
a)

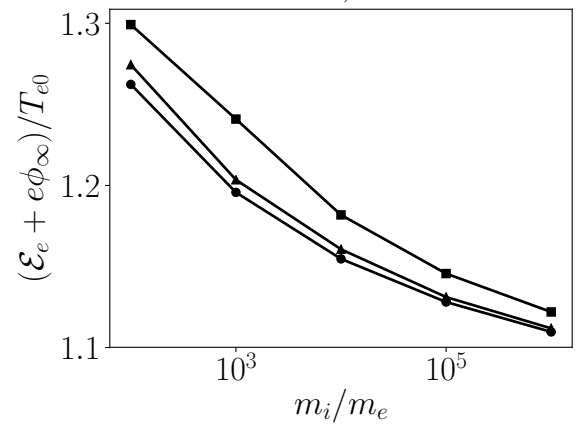

b)

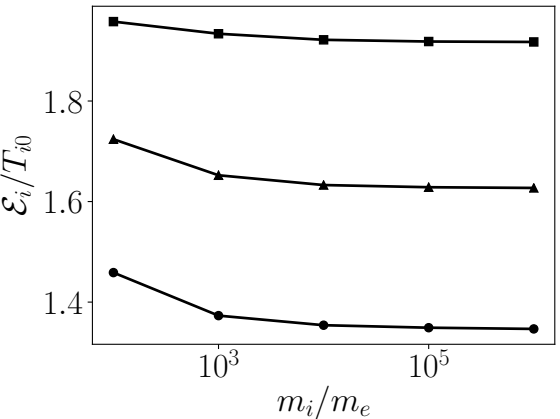

Figure 7: (a) $\mathcal{E}_{e}$ and (b) $\mathcal{E}_{i}$ of equation (31) as a function of $m_{i} / m_{e}$ for three values of $T_{i 0} / T_{e 0}=0.1(\bullet-)$, $T_{i 0} / T_{e 0}=1(\boldsymbol{\bullet})$ and $T_{i 0} / T_{e 0}=10(-\boldsymbol{\bullet})$.

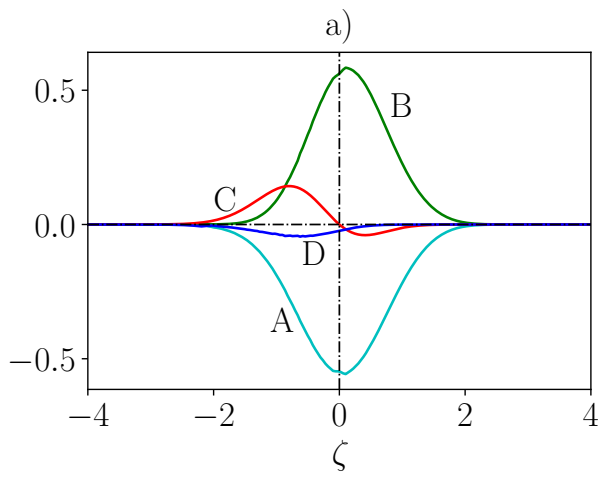

a)

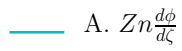

- B. $B \frac{d}{d \zeta}\left(\frac{m m u^{2}}{B}\right)$

C. C. $\left(p_{\perp}-p_{\|}\right) \frac{d \log B}{d \zeta}$

- D. $\frac{d p_{\|}}{d \zeta}$

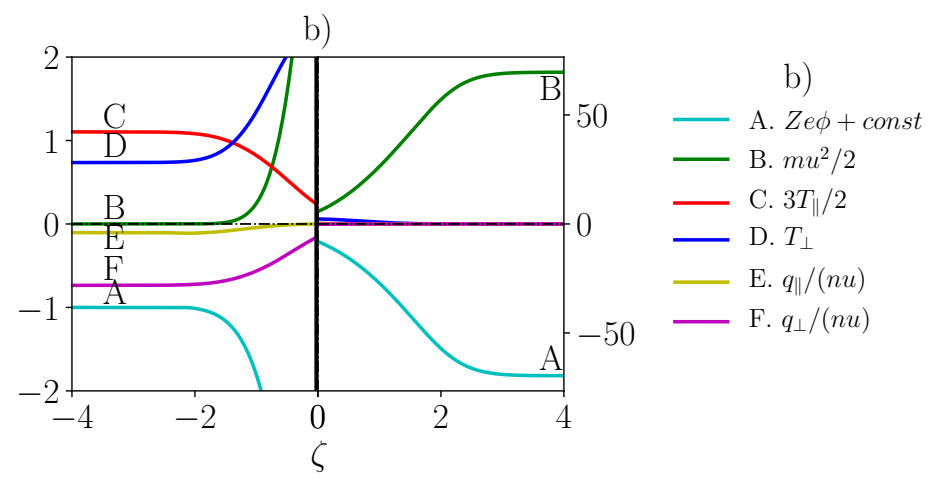

Figure 8: Relative contribution of the different terms to the equations for (a) momentum equation (28), and (b) total energy equation (29) for the cold-ion case. Units in vertical axes are arbitrary. Results are for $m_{i} / m_{e}=10^{4}$ and $T_{i 0} / T_{e 0}=0.1$.

terms of the momentum, the total energy, and the perpendicular energy equations for ions and electrons along the nozzle, thus illustrating on the dominant terms in each equation and nozzle region. Concerning plots 6(c)(d), equation (31) has been used and the constant on its right-hand side has been included into the potential energy term. This is not possible in plots 6(e)-(f) for the flows of perpendicular energy, but units have been set in order that the right-side constant in equation (30) is equal to 1.

Starting with electrons and their momentum equation, figure 6(a) shows that inertia terms are negligible, as expected. The collective MME turns out to be marginal in the whole $M N$, due to the small temperature anisotropy except at the far-downstream end. Therefore, electrons present everywhere a near perfect balance 
between the electrostatic force and the pressure gradient,

$$
e n \frac{d \phi}{d \zeta} \simeq \frac{d p_{e}}{d \zeta}
$$

Furthermore, in the convergent part of the MN, since $T_{e}$ is almost constant, and equation (32) becomes the Boltzmann relation. This is not the case in the the divergent $\mathrm{MN}$, where no simple relation among $T_{e}, n$, and $\phi$ have been found.

Regarding the conserved electron energy $\mathcal{E}_{e}$, figure $7(\mathrm{a})$ plots its dependence with $m_{i} / m_{e}$ and $T_{i 0} / T_{e 0}$. Interestingly $\mathcal{E}_{e}+e \phi_{\infty}$ is almost universal and close to $(1.2 \pm 0.1) T_{e 0}$. Once more it is worth to compare this result to the case of a conventional Debye sheath where $\mathcal{E}_{e}+e \phi_{\infty} \simeq 2 T_{e 0}$. Figure $6(\mathrm{c})$ plots the different contributions to the electron energy balance: the kinetic electron energy is negligible and the potential energy flow is the dominant contribution, which is balanced by the thermal and heat flows, with the dominance of the heat flow of parallel energy. Figure 6(e) shows that $q_{e \perp}$ and $T_{e \perp} n u_{e}$ near-balance each other except in the central region of the MN. Since the electron temperature is nearly-isotropic except in the cold downstream end, a good approximation for the electron energy balance is

$$
\frac{5}{2} T_{e}+\frac{q_{e}}{n u_{e}}-e \phi \simeq \mathcal{E}_{e}
$$

with $q_{e}=q_{e \|}+q_{e \perp}$. The error in using this approximate law, in the wide parameter range considered, is found to be below $7 \%$ in the whole MN. Regarding the dependence on $B$, the asymptotic analysis of Ramos et al. [33] for the electron population at the downstream end of the nozzle, yielded $n T_{e \|} u \propto B$ and $n T_{e \perp} u \propto B^{5 / 3}$, which is confirmed here. Additionally, it is found here that similar scaling laws apply to the parallel and perpendicular heat fluxes: $q_{e \|} \propto B$ and $q_{e \perp} \propto B^{5 / 3}$. Next Subsection further analyzes electron heat fluxes.

The behavior of the hot ion population, shown on the right plots of Fig. 6, presents interesting differences with respect to the electron one. In Fig. 6(b) for the ion momentum equation, the electrostatic force is found to be marginal for hot ions (except for a discreet contribution in the divergent region). The momentum flux gain is provided mainly by the two pressure pressure terms; in particular, the macroscopic MME is a dominant contribution in the whole MN. Also, far upstream, the magnetic mirror and the parallel pressure gradient are seen to develop sooner than ion convection. Figure $6(\mathrm{~d})$ and $(\mathrm{f})$ for the ion energy show that: the kinetic energy of ions comes mainly from the electric potential energy and the parallel thermal energy; the heat flux of parallel ion energy can be considered a second-order contribution; and the total flux of perpendicular energy $q_{i \perp}+n u_{i} T_{i \perp}$ tends to zero at both nozzle ends but is non egligible in the central region of the MN. In the cold-ion case, the response of the electrons is practically the same, but the ion response is simpler, as shown in figure 8. Now, the ion kinetic and electrostatic energies dominate totally the ion response. Figure 7(b) plots $\mathcal{E}_{i}$. There is not a good-enough approximate expression, similar to equation (33), for the ion energy. The equation

$$
\frac{1}{2} m_{i} u_{i}^{2}+\frac{3}{2} T_{i \|}+T_{i \perp}+e \phi \approx \mathcal{E}_{i}
$$

yields an error of up to $10 \%$ in the central region of the nozzle and remains below $2 \%$ for both hot- and cold-ion cases along the divergent region of the expansion. Nonetheless, equation (34) is not valid far upstream (where relative errors are of order one). 


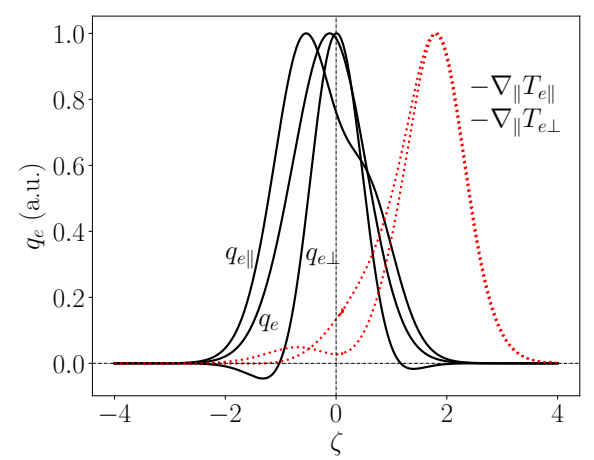

Figure 9: Normalized evolution of electron heat fluxes and gradients of temperatures along the nozzle for $m_{i} / m_{e}$ $=10^{4}$, and $T_{i 0} / T_{e 0}=10$.

\section{IV.2 Electron heat fluxes}

Using the preceding fluid equations directly, i.e. without solving the kinetic Vlasov equation, requires closure relations for the heat fluxes. Clearly, the CGL double adiabatic limit, $q_{\|}=q_{\perp}=0$ [37], does not apply. Still, ion heat fluxes are not a dominant contribution to the energy balance and could be ignored in a first approach, as in equation (34). This is not the case for the electron heat fluxes, which are further investigated in this subsection.

First, electron heat fluxes do not follow a Fourier law, as it evident from Fig. 9, which compares the shapes of the heat fluxes and the temperature gradients and shows that they are not proportional. Furthermore, the upstream and downstream electron heat fluxes satisfy

$$
\begin{gathered}
\zeta=-\infty: \quad \frac{q_{e \perp}}{n u_{e}}=-T_{e 0}, \quad \frac{q_{e}}{n u_{e}}=\frac{3}{2} T_{e 0}+e\left|\phi_{\infty}\right|, \\
\zeta \rightarrow+\infty: \quad \frac{q_{e \perp}}{n u_{e}}=0, \quad \frac{q_{e} \|}{n u_{e}} \simeq \mathcal{E}_{e} \approx 1.2 T_{e 0},
\end{gathered}
$$

so they are nonzero, in general, while the temperature gradients become null at the two MN ends. These facts indicate that a heat flux in collisionless and collisional fluids does not represent the same physics. In both fluids, the heat flux is the difference between the total and convective fluxes of total energy, Eqs. (11) and (12). In a collisional fluid, the heat flux has the simple and clear meaning expressed by the Fourier law: it develops when a temperature gradient exists and its effect is to reduce that gradient. No such meaning and effect extend to a collisionless fluid, where the heat flux is principally a mathematical entity. A physical meaning can theoretically be extracted from the third-order equations in the fluid hierarchy for the transport of $q_{e \|}$ and $q_{e \perp}$; see, for instance, Eqs. (43) and (44) of Ref. [38]. However, the complexity of these equations and the presence of next-order terms make uncertain they can unveil a clear physical interpretation of these fluxes.

Instead, in order to understand better the relevance here of the heat fluxes (i.e. the diffusive fluxes of thermal energy), Figs. 10(a)-(d) compare them with the convective thermal fluxes. First, Fig. 10(a) shows that the total flux of perpendicular energy, $q_{e \perp}+n u_{e} T_{e \perp}$, is positive all along the MN and tends to zero at both nozzle ends, although only at the downstream end both individual contributions are zero. The negative value of $q_{e \perp}$ at the two asymptotic regions is a good example of its predominantly mathematical character. In the central region of the $\mathrm{MN}$, the total flux of perpendicular energy increases as a consequence of the individual magnetic mirror effect on electrons; as a consequence $q_{e \perp}$ increases (and changes sign). Next, Fig. 10(b) compares the 

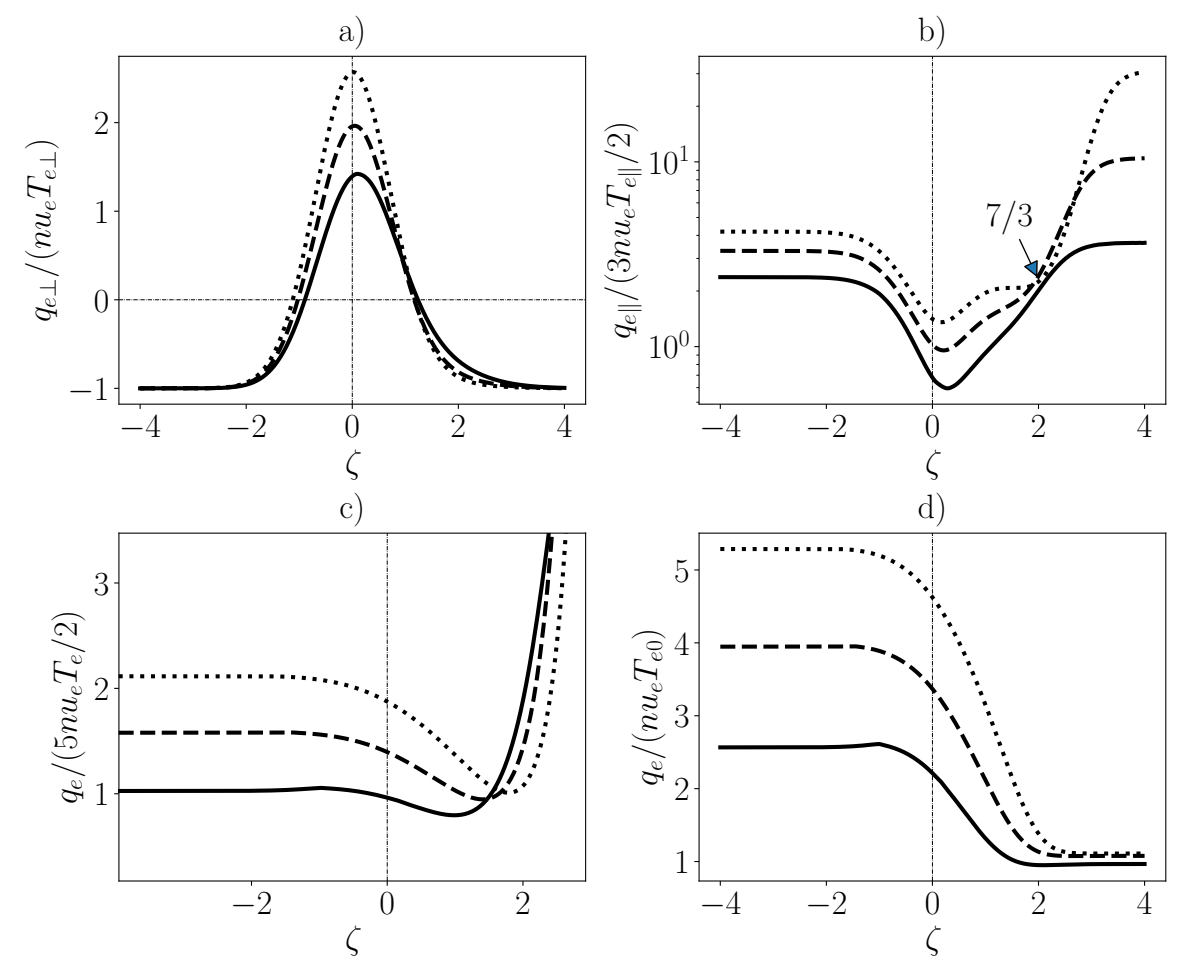

Figure 10: (a) Ratio of perpendicular heat flux versus perpendicular thermal energy flux. (b) Ratio of parallel heat flux versus parallel thermal energy flux. (c) Ratio of total heat flux versus thermal energy flux. (d) Ratio of total heat flux versus total flux. $m_{i} / m_{e}=10^{3}$ (-), $10^{4}(---), 10^{5}$ ( …), and $T_{i 0} / T_{e 0}=10$.

two parallel energy fluxes, both being positive along the whole MN. As commented above, the heat flux is about 2.5-4 times the convective flux in the convergent $\mathrm{MN}$ in spite of the quasi-constant temperature there. Then, just downstream of the nozzle throat, there is a minimum of the diffusion-to-convection ratio, which balances the magnetic mirror effect on the fluxes of perpendicular energy. Further downstream, at $\zeta \approx 2$, all curves intertwine around the value $7 / 3$, and finally the diffusion-to-convection flux ratio increases to the large asymptotic value

$$
\frac{2 q_{e \|}}{3 n u_{e} T_{e \|}} \approx 0.8 \frac{T_{e 0}}{T_{e \| \infty}}-1
$$

obtained from equation (31) and figure 7(a).

The splitting of the heat flux of the electron thermal energy into fluxes of parallel and perpendicular thermal energy could be more a disadvantage than a benefit when looking for a comprehensive macroscopic picture of the electron response. Figure 10(c) plots the diffusion-to-convection flux ratio of total thermal energy. Compared to Figs. 10(a) and (b), this ratio is monotonic down to $\zeta \sim 2$, and then grows to attain a large asymptotic value (not shown there). Figure 10(d) plots $q_{e} / n u_{e}$, non-dimensionalized with $T_{e 0}$ and confirms that the heat flow $q_{e} / B$ (which is proportional to $q_{e} / n u_{e}$ ) is monotonically decreasing. The absence of local extrema around the nozzle throat in figures 10 (c) and (d), confirms the compensation there of the magnetic mirror effects on parallel and perpendicular heat fluxes, caused by the exchange between perpendicular energy diffusive flux, $m_{e}\left\langle w_{\perp}^{2} c_{\|}\right\rangle / 2$, and parallel energy diffusive flux, $m_{e}\left\langle c_{\|}^{3}\right\rangle / 2$.

In summary, in terms of the electron energy, three regions can be identified in the MN: (1) the convergent region which is near isothermal but with a rather large heat flux, (2) the main divergent region, reaching until 
$B / B_{M} \sim 0.01$, with both $T_{e}$ and $q_{e} / B$ decreasing, and (3) the far downstream region, with $q_{e} / B$ near constant and $T_{e}$ still decreasing to its asymptotic value.

The changing behavior of $q_{e}$ along the MN and its unclear physical basis does not help to derive a closure relation for the set of electron fluid equations. Nonetheless, to end this subsection, let us comment a crude phenomenological law for $q_{e}$, which in the end is going to be equivalent to the phenomenological polytropic law, often used to analyze plasma expansions. Based on figure 10(c), let us take an average value of the diffusion-to-convection flux ratio, i.e. $q_{e} / n u_{e} T_{e}=$ const or, in vector formulation,

$$
\boldsymbol{q}_{e}=\bar{\alpha} n T_{e} \boldsymbol{u}_{e},
$$

with $\bar{\alpha}$ a constant. This is substituted in the stationary internal energy equation

$$
\nabla \cdot\left(\frac{5}{2} T_{e} n \boldsymbol{u}_{e}+\boldsymbol{q}_{e}\right)=\boldsymbol{u}_{e} \cdot \nabla p_{e}+Q_{e},
$$

where, for compactness, $Q_{e}$ groups all volumetric sources of internal energy. For the stationary, collisionless case, it is $Q=0$ and $\nabla \cdot n \boldsymbol{u}_{e}=0$, and a straightforward integration of the energy equation yields

$$
T_{e} \propto n^{\bar{\gamma}-1} \quad \text { with } \quad \bar{\gamma}=\frac{5+2 \bar{\alpha}}{3+2 \bar{\alpha}},
$$

with $\bar{\gamma}$ an effective polytropic coefficient. The adiabatic case $\bar{\alpha}=0$ corresponds to $\bar{\gamma}=5 / 3$ and, as the relative heat flux increases, $\bar{\gamma}$ decreases. For instance, the intermediate value $\alpha=7 / 2$, extracted from figure 10(c), o yields $\bar{\gamma} \approx 1.2$, a value close to some experimental evidence [26-29,39]. Implementing the crude closure (37) in the energy equation (33) yields the known law between the electric potential fall and the polytropic coefficient, [34]

$$
\frac{e\left|\phi_{\infty}\right|}{T_{e 0}} \approx \frac{\bar{\gamma}}{\bar{\gamma}-1} .
$$

This implies that $\bar{\gamma}$ depends on the mass and temperature ratios: for the cold-ion case and using equation (26) one has

$$
\bar{\gamma} \approx 1+\left(1+\ln \sqrt{\frac{m_{i}}{2 \pi m_{e}}}\right)^{-1} .
$$

In summary, in a collisionless plasma, the polytropic law (39) is equivalent to a constant diffusion-toconvection energy flux law (37). Indeed the variation of $q_{e} / n u_{e} T_{e}$ along the nozzle is equivalent to the variation of the 'local polytropic coefficient'

$$
\gamma=1+\frac{d \ln T_{e}}{d \ln n}
$$

computed in Ref. [34], for instance. The advantage of the flux-based law proposed here is that it is easily extended to a weakly collisional plasma expansion. However, the main point to stand out is that both of them are crude phenomenological closures of a collisionless fluid model and do not reproduce the real electron energy balance, mainly in the divergent MN, which generally is the region of most practical interest. This would partially explain that some experimental data requires to be matched with piecewise polytropic laws [27, 28]. In any case, these simple laws have a practical value for fluid simulations, since they provide acceptable spatial profiles of the electric potential, and the plasma density and temperatures.

\section{Summary and conclusions}

A previous work on a kinetic paraxial model of collisionless magnetized plasma, channelled by a convergentdivergent $\mathrm{MN}$, has been complemented here in several directions. One of the central studies has been on mag- 
netic mirror effects. In the fully-magnetized case, those are equally important to ion and electron particles, but macroscopically, magnetic mirror effects manifest only if temperature anisotropy is generated. Contrary to naive intuition, it has been shown that the collective mirror effect is very mild on electrons, in both convergent and divergent regions, except very far downstream (where anyway the electron pressure and temperature are residual).

On the contrary, magnetic mirror effects are shown to be strong on ions, generating a large temperature anisotropy in the ion population. Furthermore, ions change from having $T_{i \perp} / T_{i \|}>1$ in the convergent MN to $T_{i \perp} / T_{i \|} \rightarrow 0$ far downstream. Related also to the ion temperature, the comparison of the plasma response when the upstream ion VDF is monoenergetic or Maxwellian shows changes only on second and higher order velocity moments, such as $T_{i}$ and $q_{i}$.

The collisionless macroscopic (i.e. fluid) equations for ions and electrons have been analyzed using the moments of the kinetic solution. First, the ion fluid behavior is different for the cold- and hot-ion cases. While in the first case ion dynamics are dominated by the ambipolar electric field, in the second one the anisotropic pressure and the collective magnetic mirror effect have dominant roles and even ion heat fluxes are not fully negligible in the energy balance.

Since collective magnetic mirror effects are small for electrons, their momentum equation reduces to the standard equilibrium between the electric and pressure forces, but no simple relation between $p_{e}$ and $n$ (such as the Boltzmann relation) can be invoked in general. In the present collisionless case, the equations for parallel and perpendicular electron energies are the most challenging ones theoretically, since electron heat fluxes are dominant terms in them even in the regions where the electrons are practically isothermal. The electron heat flow $q_{e} / B$ is large and near-constant in the convergent nozzle and then decreases around the throat and the divergent nozzle to reach a downstream asymptotic value. In almost the whole MN, the diffusive thermal flux $q_{e}$ is larger than the convective one, $(5 / 2) T_{e} n u_{e}$, so its variation along the nozzle is related closely to the total potential fall.

The electron heat fluxes are far from fulfilling a Fourier-type law; in fact, a physical meaning for them in a collisionless fluid is uncertain. Without it, a heat flux is just the mathematical difference between the total (physical) energy flux and what is defined as the convective flux. The search of a physical basis should be further pursued analyzing the corresponding third-order electron fluid equation.

Here, in order to find an approximate closure of the fluid equations, a simple phenomenological law based on a constant diffusion-to convective thermal flux ratio has been discussed. It has been demonstrated to be equivalent to the often invoked polytropic closure, but presents the advantage of being applicable to weaklycollisional discharges too. In any case, these simple laws, although practical, do not reproduce the locally changing physics of the real collisionless discharges.

Due to their practical interest, the parametric dependence of $e\left|\phi_{\infty}\right| / T_{e 0}$ on the two free parameters of the model, the propellant mass and temperature ratios, has been assessed and good analytical approximations have been derived. Similar laws have been obtained for the final beam velocity and parallel temperatures of ions and electrons. Finally and motivated by a previous work on the plasma discharge in a convergent MN followed by a Debye sheath and a wall, the similarities and differences between the electron confinement and electric current control by a very thin sheath and by the semi-infinite divergent region of a MN have been discussed.

The goal of the present paraxial MN model has been to understand fundamental kinetic aspects of a

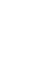


collisionless plasma discharge and their macroscopic manifestation. The implementation of the new findings in the $2 \mathrm{D}$ or $3 \mathrm{D}$ electron fluid models used, for instance, by the codes DIMAGNO [13], FUMAGNO [24] or EP2PLUS [40,41] should improve the simulations of plasma expansions in real configurations such as those of space electric thrusters.

\section{Acknowledgments}

This work was supported by the Spanish R \& D National Plan (Grant No. PN ESP2016-75887) and by the European Union Horizon 2020 project MINOTOR, that has received funding from the research and innovation program under grant agreement No 730028. The authors thanks Dr. Jesús Ramos for his interesting comments on this work.

\section{${ }_{475}$ Appendix A Evolution of the velocity distribution functions}

In the parametric plane $(\mu, E)$, the VDF of each species, $f$, is constant to first order along the $\zeta$ direction. This constant propagation of $f$ from the upstream plasma reservoir is only interrupted by the turning points of the axial velocity, which are given by equation (5), that is

$$
L_{\zeta}: \quad E(\mu ; \zeta)=Z e \phi(\zeta)+\mu B(\zeta)
$$

This equation constitutes a family of straight lines in the $(\mu, E)$ plane, with $\zeta$ as parameter. For each location $\zeta$, the region locally apt for having particles is the one above the corresponding $L_{\zeta}$. Far upstream and downstream, the lines are horizontal, while at the MN throat, the line has maximum slope, $B_{M}$ :

$$
\begin{array}{ll}
L_{0}: & E(\mu)=0, \\
L_{M}: & E(\mu)=B_{M} \mu+Z e \phi_{M}, \\
L_{\infty}: & E(\mu)=Z e \phi_{\infty} .
\end{array}
$$

These three lines, together with the envelope $\Sigma$ of family equations (43), delimit the regions of phase space of free, reflected, and doubly-trapped particles. Figure 11 displays these lines for electrons $(Z=-1)$ and ions $(Z=1)$ in the cold ions case. There are four distinct electron regions in the $(\mu, E)$ plane, separated by $L_{\infty}, L_{M}$ and $\Sigma$. Region A contains the high-energy, low- $\mu$ electrons that manage to escape the MN, i.e., free electrons, for all values of $\zeta$. Region B has reflected electrons initially, and then becomes energetically forbidden downstream as $\zeta$ increases. Region $\mathrm{C}$ has reflected electrons initially, then becomes forbidden, and then allowed again but empty. Finally, the special region D is delimited by the envelope $\Sigma$, formed by line of equation (43) in the divergent part of the MN. This region transitions from having reflected electrons, to being forbidden, to having doubly-trapped electrons, to being forbidden again. It is the only region of the parametric plane that can host doubly-trapped electrons. In the case of ions, $\Sigma$ is the relevant boundary for free (region A). To its right (region C), reflected ions exist initially, then this region becomes forbidden, and then available and empty again..

In order to illustrate the evolution of the ion and electron VDFs along the magnetic nozzle and complement the discussion in the main text, Figure 12 plots three examples in the $\left(w_{\|}, w_{\perp}\right)$ velocity plane. The first column corresponds to electrons (for the cold-ion case). The second column corresponds to cold Maxwellian ions (a 

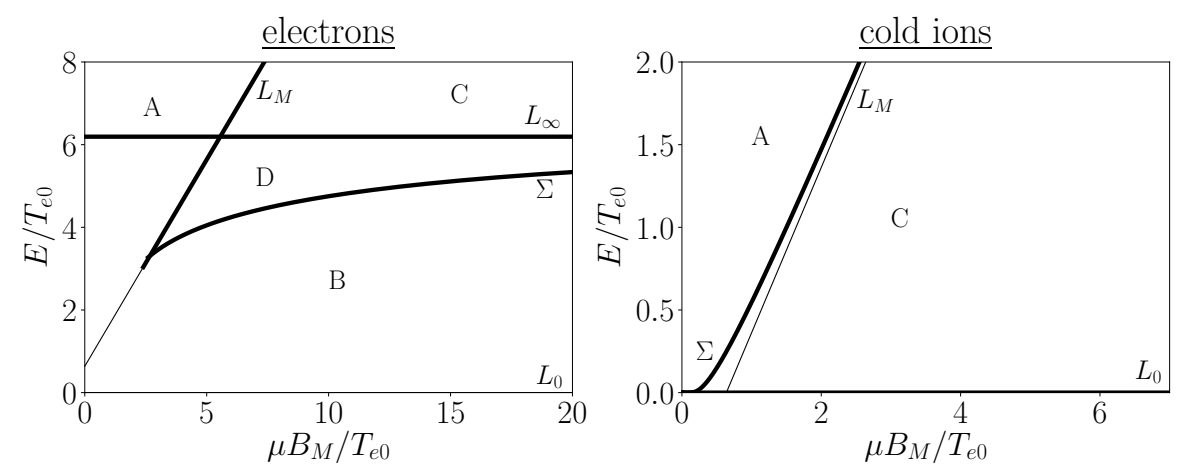

Figure 11: Regions in the $(\mu, E)$ plane for electrons and ions in the cold ion case, showing the lines $L_{0}$, $L_{M}$, $L_{\infty}$ and the envelope $\Sigma$. The thicker lines delimit regions where different types of particles are allowed and correspond to: A, free particles; B (only electrons), reflected low energy particles, then forbidden; $C$, reflected high energy particles, then forbidden, then empty; D (only electrons), reflected particles, then forbidden, then doubly-trapped particles, then forbidden again.

Maxwellian $f_{i 0}^{+}$with $T_{i 0} / T_{e 0}=0.1$ ) and the third one to hot ions (a Maxwellian $f_{i 0}^{+}$with $T_{i 0} / T_{e 0}=10$ ). For additional illustration, the thick black rings on the two last columns correspond roughly to a monoenergetic $f_{i 0}^{+}$.

The three lines $L_{0}, L_{M}$ and $L_{\infty}$ of equations (44), (45), and (46) become the following conical sections in the velocity $\left(v_{\|}, v_{\perp}\right)$ plane:

$$
\begin{aligned}
L_{0}: & w_{\|}^{2}+w_{\perp}^{2}=-\frac{2 Z e}{m_{\alpha}} \phi(\zeta), \\
L_{M}: & w_{\|}^{2}-\left(\frac{B_{M}}{B(\zeta)}-1\right) w_{\perp}^{2}=\frac{2 e Z_{\alpha}}{m_{\alpha}}\left[\phi_{M}-\phi(\zeta)\right], \\
L_{\infty}: & w_{\|}^{2}+w_{\perp}^{2}=-\frac{2 Z e}{m_{\alpha}}\left[\phi(\zeta)-\phi_{\infty}\right] .
\end{aligned}
$$

These lines are plotted in Figure 12 as a dotted semi-circle, dash-and-dot hyperbola, and dashed circle, respectively. The envelope $\Sigma$ is the free boundary of the coloured region, joining with the other curves smoothly. Observe that $L_{M}$ for ions and electrons are conjugated hyperbolas, and that these hyperbolas become straight lines at $\zeta=0$, position where they swap the major axis direction. In all plots, those particles on the $w_{\|}>0$ side of the plane that do not have a corresponding image particle on the $w_{\|}<0$ side are free particles; regions where particles exist for both $w_{\|}>0$ and $w_{\|}<0$ represent reflected or doubly-trapped particles.

In the case of electrons (first column in the Figure), only $L_{M}$ and $L_{\infty}$ shape the regions of the VDF. Electrons within the $L_{\infty}$ semicircle cannot reach the downstream end of the MN, and electrons above the hyperbola $L_{M}$ cannot reach the nozzle throat. Therefore, only electrons above $L_{\infty}$ and to the right of $L_{M}$ are free electrons. For $\zeta<0$ the rest of them are reflected electrons. For $\zeta>0$, those below $L_{\infty}$ and $L_{M}$ are reflected, while those between these two lines are doubly-trapped ones.

In the case of ions (two last columns), only $L_{0}$ and $\Sigma$ (which approaches $L_{M}$ in most plots) play a role as indicated in the analysis of the $(\mu, E)$ plane. The $L_{0}$ semi-circle delimits a region below which no ions exist. For $\zeta<0$, the forward-moving ions located above $\Sigma$ cannot reach the throat and are reflected back; below $\Sigma$ and above $L_{0}$, most of the ions are free and the region $w_{\|}<0$ is almost empty. For $\zeta>0$, all ions are free ions, and are located to the right of $\Sigma$ and above $L_{0}$. Far upstream essentially all ions are reflected and the ion VDF is Maxwellian. As the expansion begins, an empty region appears (and correspondingly, the free ions can be 

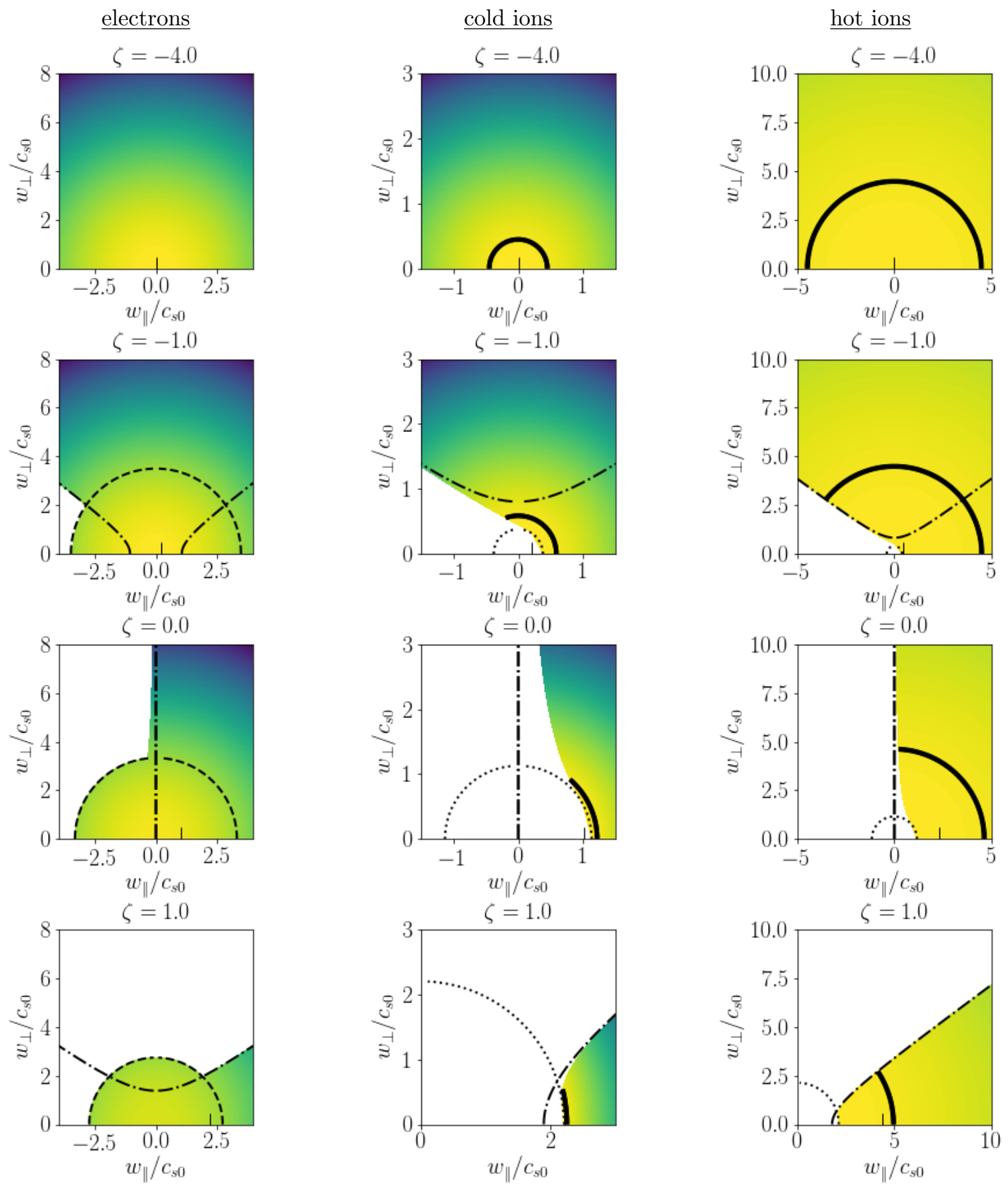

Figure 12: Electron and ion VDFs in the velocity plane at four different spatial locations. First column is for electrons in the cold-ion case. Second and Third columns are for a Maxwellian $f_{i 0}^{+}$with $T_{i 0} / T_{e 0}=10$ and 0.10 , respectively; the thick solid line is for the equivalent monoenergetic case of $f_{i 0}^{+}$. The mass ratio is $m_{i} / m_{e}=10^{4}$; $c_{s 0}=\sqrt{T_{e 0} / m_{i}}$. Dotted lines are for $L_{0}$, dash-and-dot ones for $L_{M}$, and dashed ones for $L_{\infty}$. The vertical tick in the $x$-axis is the local value of the macroscopic velocity $u_{i}$. Observe that the $w_{\|}$axis is shifted in this last row.

identified).

At the throat and beyond all ions are free ions. In the divergent side, the empty region grows further, and the hyperbola of equation (48) becomes more eccentric until far downstream only ions with $w_{\perp} \rightarrow 0$ exist.

The different behavior of $T_{i \perp}$ at the throat $M$ for hot- and cold- ions, shown in figure $3(\mathrm{f})$, can be 
explained in view of the ion VDF of figure 12. At the throat $(\zeta=0)$, the ion VDF would extend the semiplane $w_{\|}>0$ and yield $T_{i \perp}=T_{i 0}$, were it not for the empty region below the semi-circle $L_{0}$ and the arc of envelope $\Sigma$. The relevance of this empty region in the integral definition of $T_{i \perp}$ is small for hot-ions but large for cold-ions, leading to the observed $T_{i \perp} \simeq T_{i 0}$ in the hot case and $T_{i \perp} \simeq 3 T_{i 0}$ in the cold case. 
[1] Mario Merino and Eduardo Ahedo. Magnetic nozzles for space plasma thrusters. In J. Leon Shohet, editor, Encyclopedia of Plasma Technology, volume 2, pages 1329-1351. Taylor and Francis, 2016.

[2] Kazunori Takahashi, Christine Charles, Rod Boswell, and Akira Ando. Performance improvement of a permanent magnet helicon plasma thruster. Journal of Physics D: Applied Physics, 46(35):352001, 2013.

[3] R. Winglee, T. Ziemba, L. Giersch, J. Prager, J. Carscadden, and B.R. Roberson. Simulation and laboratory validation of magnetic nozzle effects for the high power helicon thruster. Physics of Plasmas, 14(6):063501, 2007.

[4] Shunjiro Shinohara, Hiroyuki Nishida, Takao Tanikawa, Tohru Hada, Ikkoh Funaki, and Konstantin P Shamrai. Development of electrodeless plasma thrusters with high-density helicon plasma sources. IEEE Transactions on Plasma Science, 42(5):1245-1254, 2014.

[5] J Navarro-Cavallé, M Wijnen, P Fajardo, and E Ahedo. Experimental characterization of a 1 kw helicon plasma thruster. Vacuum, 149:69-73, 2018.

[6] Julien Jarrige, Paul-Quentin Elias, Félix Cannat, and Denis Packan. Characterization of a coaxial ecr plasma thruster. In 44th AIAA Plasmadynamics and Lasers Conference, San Diego, 2013.

[7] A.D. Kodys and E.Y. Choueiri. A critical review of the state-of-the-art in the performance of applied-field magnetoplasmadynamic thrusters. In 41st AIAA Joint Propulsion Conference and Exhibit, volume 4247, 2005.

[8] F.R.C. Diaz, J.P. Squire, R.D. Bengtson, B.N. Breizman, F.W. Baity, and M.D. Carter. The physics and engineering of the VASIMR engine. In 36th AIAA/ASME/SAE/ASEE Joint Propulsion Conference 6 Exhibit, AIAA 2000-3756, 2000.

[9] M.A. Lieberman and A.J. Lichtenberg. Principles of plasma discharges and materials processing. John Wiley and Sons, Hoboken, NJ, 2005.

[10] B Albertazzi, A Ciardi, M Nakatsutsumi, T Vinci, J Béard, R Bonito, J Billette, M Borghesi, Z Burkley, SN Chen, et al. Laboratory formation of a scaled protostellar jet by coaligned poloidal magnetic field. Science, 346(6207):325-328, 2014.

[11] D.L. Chubb. Fully ionized quasi-one-dimensional magnetic nozzle flow. AIAA Journal, 10(2):113-114, 1972.

[12] A.V. Arefiev and B.N. Breizman. Ambipolar acceleration of ions in a magnetic nozzle. Physics of Plasmas, 15(4):042109, 2008.

[13] Eduardo Ahedo and Mario Merino. Two-dimensional supersonic plasma acceleration in a magnetic nozzle. Physics of Plasmas, 17(7):073501, 2010.

[14] Mario Merino and Eduardo Ahedo. Plasma detachment in a propulsive magnetic nozzle via ion demagnetization. Plasma Sources Science and Technology, 23(3):032001, 2014. 
[15] Mario Merino and Eduardo Ahedo. Effect of the plasma-induced magnetic field on a magnetic nozzle. Plasma Sources Science and Technology, 25(4):045012, 2016.

[16] Thomas M York, Barry A Jacoby, and Pavlos Mikellides. Plasma flow processes within magnetic nozzle configurations. Journal of Propulsion and Power, 8(5):1023-1030, 1992.

[17] K. Kuriki and O. Okada. Experimental study of a plasma flow in a magnetic nozzle. Physics of Fluids, $13(9): 2262,1970$.

[18] M. Inutake, A. Ando, K. Hattori, H. Tobari, and T. Yagai. Characteristics of a supersonic plasma flow in a magnetic nozzle. J. Plasma Fusion Res., 78(12):1352-1360, 2002.

[19] C.A. Deline, R.D. Bengtson, B.N. Breizman, M.R. Tushentsov, J.E. Jones, D.G. Chavers, C.C. Dobson, and B.M. Schuettpelz. Plume detachment from a magnetic nozzle. Physics of Plasmas, 16(3):033502, 2009.

[20] B.W. Longmier, E.A. Bering, M.D. Carter, L.D. Cassady, W.J. Chancery, F.R.C. Díaz, T.W. Glover, N. Hershkowitz, A.V. Ilin, G.E. McCaskill, et al. Ambipolar ion acceleration in an expanding magnetic nozzle. Plasma Sources Science and Technology, 20:015007, 2011.

[21] Rod W Boswell, Kazunori Takahashi, Christine Charles, and Igor D Kaganovich. Non-local electron energy probability function in a plasma expanding along a magnetic nozzle. Frontiers in Physics, 3:14, 2015.

[22] C.S. Olsen, M.G. Ballenger, M.D. Carter, F.R. Chang Diaz, M. Giambusso, T.W. Glover, A.V. Ilin, J.P. Squire, B.W. Longmier, E.A. Bering, and P.A. Cloutier. Investigation of plasma detachment from a 570 magnetic nozzle in the plume of the vx-200 magnetoplasma thruster. Plasma Science, IEEE Transactions on, 43(1):252-268, 2015.

[23] J. Jarrige, P.Q. Elias, F. Cannat, and D. Packan. Performance comparison of an ECR plasma thruster using argon and xenon as propellant gas. In 33rd International Electric Propulsion Conference, Paper 2013-420, Washington D.C., October 7-10, 2013. Electric Rocket Propulsion Society, Fairview Park, OH.

[24] Mario Merino and Eduardo Ahedo. Fully magnetized plasma flow in a magnetic nozzle. Physics of Plasmas, 23(2):023506, 2016.

[25] Mario Merino and Eduardo Ahedo. Influence of electron and ion thermodynamics on the magnetic nozzle plasma expansion. IEEE Transactions on Plasma Science, 43(1):244-251, Jan 2015.

[26] JM Little and EY Choueiri. Electron cooling in a magnetically expanding plasma. Physical Review Letters, 580 117(22):225003, 2016.

[27] S Correyero, J Jarrige, D Packan, and E Ahedo. Plasma beam characterization along the magnetic nozzle of an ECR thruster. Plasma Sources Science and Technology, 28(9):095004, 2019.

[28] June Young Kim, KS Chung, Seongcheol Kim, Jong Hyeon Ryu, Kyoung-Jae Chung, and YS Hwang. Thermodynamics of a magnetically expanding plasma with isothermally behaving confined electrons. New 585 Journal of Physics, 20(6):063033, 2018. 
[29] T Lafleur, F Cannat, J Jarrige, PQ Elias, and D Packan. Electron dynamics and ion acceleration in expanding-plasma thrusters. Plasma Sources Science and Technology, 24(6):065013, 2015.

[30] Manuel Martínez-Sánchez, Jaume Navarro-Cavallé, and Eduardo Ahedo. Electron cooling and finite potential drop in a magnetized plasma expansion. Physics of Plasmas, 22(5):053501, 2015.

[31] Gonzalo Sánchez-Arriaga, Jiewei Zhou, E Ahedo, Manuel Martínez-Sánchez, and Jesús José Ramos. Kinetic features and non-stationary electron trapping in paraxial magnetic nozzles. Plasma Sources Science and Technology, 27(3):035002, 2018.

[32] J. Zhou, G. Sánchez-Arriaga, E. Ahedo, M. Martínez-Sánchez, and J.J. Ramos. Collisional effects in non-stationary plasmas expansions along convergent-divergent magnetic nozzles. In Space Propulsion Conference 2018, number 00332, Seville, Spain, 2018. Association Aéronautique et Astronautique de France.

[33] Jesús Ramos, Mario Merino, and Eduardo Ahedo. Three dimensional fluid-kinetic model of a magnetically guided plasma jet. Physics of Plasmas, 25(6):061206, 2018.

[34] Mario Merino, Javier Mauriño, and Eduardo Ahedo. Kinetic electron model for plasma thruster plumes. Plasma Sources Science and Technology, 27(3):035013, 2018.

[35] Manuel Martínez-Sánchez and Eduardo Ahedo. Magnetic mirror effects on a collisionless plasma in a convergent geometry. Physics of Plasmas, 18(3):033509, 2011.

[36] B.N. Breizman, M.R. Tushentsov, and A.V. Arefiev. Magnetic nozzle and plasma detachment model for a steady-state flow. Physics of Plasmas, 15:057103, 2008.

[37] G.F. Chew, M.L. Goldberger, and F.E. Low. The boltzmann equation and the one-fluid hydromagnetic equations in the absence of particle collisions. Proceedings of the Royal Society of London A, 236:112-118, 1956.

[38] J.J. Ramos. Dynamic evolution of the heat fluxes in a collisionless magnetized plasma. Physics of Plasmas, 10:3601, 2003.

[39] June Young Kim, Jae Young Jang, KS Chung, Kyoung-Jae Chung, and YS Hwang. Time-dependent kinetic analysis of trapped electrons in a magnetically expanding plasma. Plasma Sources Science and Technology, 28(07LT01), 2019.

[40] Filippo Cichocki, Adrián Domínguez-Vázquez, Mario Merino, and Eduardo Ahedo. Hybrid 3D model for the interaction of plasma thruster plumes with nearby objects. Plasma Sources Science and Technology, 26(12):125008, 2017.

[41] Filippo Cichocki, Mario Merino, and Eduardo Ahedo. A 3d electron fluid model to study magnetic field effects on an expanding plasma thruster plume. In Space Propulsion Conference 2018, number 00295, Seville, Spain, May 14-18, 2018. Association Aéronautique et Astronautique de France. 